\title{
An 'Internet of Things' enabled dynamic optimization method for smart vehicles and logistics tasks
}

Sichao Liu, Yingfeng Zhang, Yang Liu, Lihui Wang and Xi Vincent Wang

The self-archived postprint version of this journal article is available at Linköping University Institutional Repository (DiVA):

http:// urn.kb.se/ resolve?urn=urn:nbn:se:liu:diva-154906

N.B.: When citing this work, cite the original publication.

Liu, S., Zhang, Y., Liu, Y., Wang, L., Wang, Xi V., (2019), An 'Internet of Things' enabled dynamic optimization method for smart vehicles and logistics tasks, J ournal of Cleaner Production, 215, 806820. https:// doi.org/ 10.1016/j.jclepro.2018.12.254

Original publication available at:

https:// doi.org/ 10.1016/j.jclepro.2018.12.254

Copyright: Elsevier

http:// www.elsevier.com/

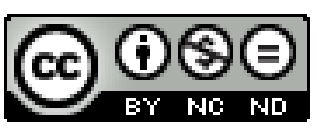




\title{
An 'Internet of Things' enabled Dynamic Optimization Method for
}

\section{Smart Vehicles and Logistics Tasks}

\author{
Sichao Liu ${ }^{\text {a }}$, Yingfeng Zhang ${ }^{\text {b, }}$, Yang Liu ${ }^{\mathrm{c}, \mathrm{d}, * \text {, Lihui Wang }}{ }^{\mathrm{a}}$, Xi Vincent Wang ${ }^{\mathrm{a}}$
}

${ }^{a}$ Department of Production Engineering, KTH Royal Institute of Technology, Stockholm, Sweden

${ }^{\mathrm{b}}$ Key Laboratory of Contemporary Design and Integrated Manufacturing Technology, Ministry of

Education, Northwestern Polytechnical University, Shaanxi, P. R. China, 710072

${ }^{c}$ Department of Management and Engineering, Linköping University, SE-581 83 Linköping, Sweden

${ }^{\mathrm{d}}$ Department of Production, University of Vaasa, 65200 Vaasa, Finland

*Corresponding author. E-mail addresses: zhangyf@nwpu.edu.cn (Y. Zhang), yang.liu@liu.se (Y. Liu)

Abstract: Centralized and one-way logistics services and the lack of real-time information of logistics resources are common in the logistics industry. This has resulted in the increased logistics cost, energy consumption, logistics resources consumption, and the decreased loading rate. Therefore, it is difficult to achieve efficient, sustainable, and green logistics services with dramatically increasing logistics demands. To deal with such challenges, a real-time information-driven dynamic optimization strategy for smart vehicles and logistics tasks towards green logistics is proposed. Firstly, an 'Internet of Things'-enabled real-time status sensing model of logistics vehicles is developed. It enables the vehicles to obtain and transmit real-time information to the dynamic distribution center, which manages value-added logistics information. Then, such information can be shared among logistics companies. A dynamic optimization method for smart vehicles and logistics tasks is developed to optimize logistics resources, and achieve a sustainable balance between economic, environmental, and social objectives. Finally, a case study is carried out to demonstrate the effectiveness of the proposed optimization method. The results show that it contributes to reducing logistics cost and fuel consumption, improving vehicles' utilization rate, and achieving real-time logistics services with high efficiency.

Keywords: Internet of Things; Green logistics; Dynamic optimization; Real-time information

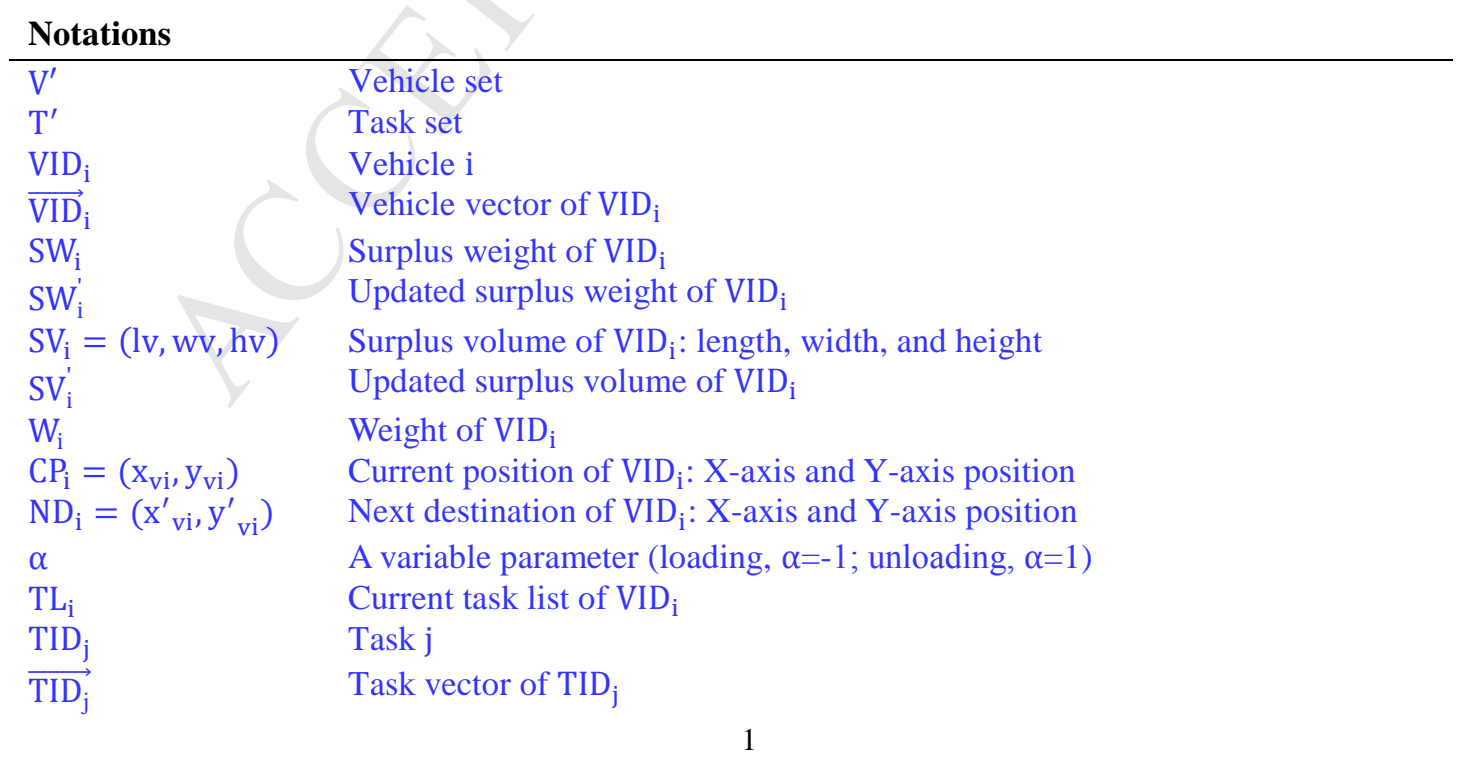




\begin{tabular}{|c|c|}
\hline$\overline{W_{j}}$ & Weight of TID $_{j}$ LI ILE IVIAIVUNCIIII \\
\hline$V_{j}=(l t, w t, h t)$ & Volume of TID $_{\mathrm{j}}$ : length, width, and height \\
\hline $\mathrm{CP}_{\mathrm{j}}=\left(\mathrm{x}_{\mathrm{tj}}, \mathrm{y}_{\mathrm{tj}}\right)$ & Current position of $\operatorname{TID}_{\mathrm{j}}: \mathrm{X}$-axis and $\mathrm{Y}$-axis position \\
\hline$N D_{j}=\left(x_{t j}^{\prime}, y_{t j}^{\prime}\right)$ & Next destination of $\mathrm{TID}_{\mathrm{j}}: \mathrm{X}$-axis and $\mathrm{Y}$-axis position \\
\hline $\mathrm{T}_{\mathrm{j}}$ & Time of delay of $\operatorname{TID}_{j}$ \\
\hline $\mathrm{dt}_{\mathrm{j}}$ & Allowed time of delay of $\mathrm{TID}_{\mathrm{j}}$ on the task lists \\
\hline $\mathrm{w}_{\mathrm{ij}}$ & Delayed penalty parameter of the $\mathrm{j}$-th task on $\operatorname{VID}_{\mathrm{i}}$ \\
\hline$f\left(V^{\prime} D_{i}\right)$ & Angle between $\operatorname{VID}_{\mathrm{i}}$ and $\mathrm{TID}_{\mathrm{j}}$ \\
\hline d & Distance from the midpoint of $\left|\overrightarrow{\mathrm{CP}_{\mathrm{i}} \mathrm{ND}_{\mathrm{i}}}\right|$ to the midpoint of $\left|\overrightarrow{\mathrm{CP}_{\mathrm{j}} \mathrm{ND}_{\mathrm{j}}}\right|$ \\
\hline $\mathrm{D}$ & Distance of $\operatorname{VID}_{i}$ finishing $\operatorname{TID}_{j}$ \\
\hline $\mathrm{O}(\mathrm{R})$ & Center of the circle \\
\hline $\mathrm{C}$ & Total logistics cost of finishing all the tasks \\
\hline $\mathrm{C}_{0}$ & Cost of finishing all the tasks in the centralized and one-way logistics service \\
\hline $\mathrm{C}_{1}$ & Delivery cost of vehicles finishing all the tasks, including the accepted task \\
\hline $\mathrm{u}_{1}$ & $\operatorname{TID}_{\mathrm{j}}$ \\
\hline $\mathrm{C}_{2}$ & Cost of vehicles finishing the tasks on the task lists \\
\hline $\mathrm{C}_{3}$ & Delayed delivery penalty cost caused by accepting TID $_{j}$ \\
\hline $\mathrm{VC}_{\mathrm{i}}$ & Cost per kilometer of $\operatorname{VID}_{\mathrm{i}}$ \\
\hline & Total distribution distance of finishing all the tasks after receiving \\
\hline $\mathrm{L}_{\mathrm{ij}}$ & $\mathrm{TID}_{\mathrm{j}}$ \\
\hline $\mathrm{L}_{\mathrm{i}}$ & Distance of $\operatorname{VID}_{\mathrm{i}}$ \\
\hline $\mathrm{L}_{\mathrm{j}}$ & Efficient distance of $\operatorname{TID}_{j}$ \\
\hline $\mathrm{L}_{\mathrm{i}}^{\prime}$ & Total distance of $\operatorname{VID}_{\mathrm{i}}$ finishing the tasks on the task lists \\
\hline$t_{j}$ & Time of delay of $\operatorname{TID}_{\mathrm{j}}$ on the task lists. \\
\hline $\mathrm{L}_{j}^{\prime}$ & Distribution distance of $\operatorname{TID}_{j}$ \\
\hline$v^{\prime}$ & Average effective loading rate of vehicles \\
\hline$\overline{\mathrm{V}}_{\mathrm{i}}$ & Real-time loading volume of $\operatorname{VID}_{\mathrm{i}}$ \\
\hline$\overline{\mathrm{L}}_{\mathrm{i}}$ & Real-time distance of $\mathrm{VID}_{\mathrm{i}}$ \\
\hline W' & Average effective weight of vehicles \\
\hline$\overline{\mathrm{W}}_{\mathrm{i}}$ & Real-time weight of $\operatorname{VID}_{\mathrm{i}}$ \\
\hline $\mathrm{R}$ & Average loading rate of vehicles \\
\hline TFC & Total fuel consumption \\
\hline $\mathrm{FC}_{\mathrm{i}}$ & Fuel consumption per kilometer of $\operatorname{VID}_{i}$ \\
\hline$\overline{\mathrm{FC}}$ & Average fuel consumption per kilometer \\
\hline$C^{\prime}$ & Rate of saving cost \\
\hline$\overline{\mathrm{R}}$ & Utilization rate of vehicles \\
\hline $\mathrm{N}$ & Number of vehicles needed in the current method \\
\hline$\overline{\mathrm{N}}$ & Number of vehicles needed in the proposed method \\
\hline
\end{tabular}

\section{Introduction}

Green logistics is the study of practices that aims to reduce the environmental externalities that are mainly related to the environmental issues of logistics operations and therefore develop a sustainable balance among economic, environmental and social objectives (Green logistics, 2010; Chhabra et al., 2017). Green logistics is an important trend of development and an efficient strategy in practice to address the challenges faced by the logistics industry such as dramatically increasing logistics demands, requirements in environmental protection, and high-efficiency logistics services (Dekker et al., 2012; He et al., 2017; Zaman and Shamsuddin, 2017; Rose et al., 2018). Currently, the typical challenges that logistics industry faces are composed of the rapid increase of logistics demands, the lack of real-time information of logistics resources and the sharing of information, the low-efficiency management and allocation of logistics resources, and high requirements of logistics services in cost 

commerce, online shopping, and transportation services in the last decades led to the scattered and exploding logistics demands characterized by randomness and unpredictability (European E-commerce Report, 2017; Pålsson et al., 2017). This contributes to the improvement of employment and the promotion of the logistics industry. It also results in many problems in the logistics industry such as the environmental issues and the increase of logistics costs. The environmental issues like fuel consumption and air pollution result from the large and low-efficiency logistics transportation. Serious competition among logistics enterprises also leads to the increase of logistics costs in terms of the economic and environmental aspects.

In addition, the lack of real-time information of logistics resources makes it difficult to achieve green and cost-effective logistics services (Muñoz-Villamizar et al., 2018). A good example of this is that a centralized and one-way logistics service pattern is widely adopted by logistics companies in practice, especially in small and medium-sized enterprises (Langevin et al., 1996). Logistics enterprises in a certain region intensively assign tasks to vehicles only considering destinations of tasks, and in most cases, vehicles finish logistics delivery services in the one-way. In this case, real-time information of logistics resources is often ignored and new logistics tasks cannot be sensed. Therefore, it is common that vehicles are in low loading rates in the round-trip and even empty running, which leads to the low average loading of vehicles and the increase in the number of vehicles needed (Crainic et al., 2004). Meanwhile, the sharing of information among logistics enterprises, vehicles, and tasks is also a major bottleneck of achieving real-time information-driven dynamic optimization of logistics resources (Vieira et al., 2015). Logistics enterprises, in particular, small and medium-sized enterprises, are unwilling to share the information of logistics resources because of the fierce competition and the issues of profit sharing. Thus, the real-time status of logistics resources such as the vehicle load and new logistics tasks cannot be shared. Therefore, the lack of real-time information of logistics resources and the sharing of information leads to the waste of logistics resources and the increase of logistics costs. The optimal allocation of logistics resources and the efficient decision-making of logistics services cannot also be achieved (Sheu, 2006). These issues related to the delivery and transportation of tasks, for example, the low loading rates of vehicles and the lack of technologies for exchanging data, have challenged the effectiveness of city logistics services (Muñoz-Villamizar et al., 2018). The management of logistics resources and the routing optimization of logistics transportation are also important issues faced by the logistics industry (Lai et al., 2012; Cattaruzza et al., 2017). The high cost of advanced information technologies applied in the logistics industry makes the efficient management of logistics resources difficult for small and medium-sized enterprises. This is an impact factor of leading to the increase of logistics costs and the issues of management of logistics resources such as the visualization of logistics resources. Routing optimization of vehicles can provide the optimal path for drivers and contribute to the improvement of efficiency of logistics. However, the lack of vehicle routing optimization in the logistics industry often leads to the increase in the total distribution distance of vehicles (Niu et al., 2018). Further, this results in the increase of logistics costs and energy consumption, and even the delivery tardiness of tasks. For example, when exceptions occur in logistics distribution activities such as traffic congestion and new tasks, this could lead to the increase of logistics costs and delivery tardiness.

Therefore, how to achieve green logistics transportation and services is a hot issue for the supply chain management and logistics industry. Growing attention was given to this issue throughout the 

storage, inventory management, materials handling, and all the related information processing (McKinnon et al., 2015). As many researches are ongoing, we do not cover every aspect, but focus on the real-time optimization and handling of new tasks for vehicles who are delivering the tasks on them. However, logistics distribution systems have become increasingly complex because of the increases in logistics demands, government regulations, traffic congestion, the requirement of high-frequency logistics deliveries, and environmental issues (Vieira et al., 2015). The state-of-the-art literature was surveyed in green logistics such as green logistics transportation (Fahimnia et al., 2015; Masson et al., 2017), the optimal allocation of logistics resources (Powell, 1986; Sheu, 2006), vehicle routing optimization services (Bramel and Simchi-Levi, 1995; Chen et al., 2006; Kim et al., 2015; Soleimani et al., 2017), and green supply chain management (Lai and Wong, 2012; Fahimnia et al., 2015; Khan et al., 2018). The optimal allocation of logistics resources and vehicles routing problem (VRP) play a vital role in the logistics delivery. For example, research on the allocation methodologies of logistics resources for quick response to the variety of customer order demands changing in short-term time intervals was of vital importance to efficient demand-responsive city logistics distribution operations (Poon et al., 2006). Research in terms of incorporating real-time logistics information into green logistics was also limited in the existing literature. In addition, the government in taxation, financial incentives, regulation, infrastructure, and land-use planning policies has an important effect on promoting the development of green logistics (McKinnon, 2010). With the growing interest in the issues of green logistics, the government takes actions in policies, financial support, guidance, and strategies to balance economic, environmental, and social objectives towards sustainable logistics (Srivastava, 2007; Rehman et al., 2016; Zaman and Shamsuddin, 2017). From logistics to the green supply chain, green logistics is one of the vital sub-components of green supply chain management process (Sarkis, 2003; Isaksson et al., 2011), which contributes to achieving a more sustainable balance between energy demand, environment, and economic health (Zaman and Shamsuddin, 2017). For example, improving economic efficiency and competitiveness is helpful to reduce environmental concerns from green growth agenda (Rao and Holt, 2005).

Despite significant progress achieved in green logistics, major challenges still exist in the realtime information-driven logistics services and the optimal allocation of logistics resources as follows.

(1) The lack of real-time, accurate and consistent information of logistics resources and the sharing of information is a major bottleneck to achieve modern green logistics. Meanwhile, new logistics tasks cannot be sensed and handled in real time. Therefore, a typical challenge is how to sense and obtain real-time information of logistics resources.

(2) The current practice where a centralized and one-way service pattern is widely used by logistics companies has many typical problems like the low-efficiency management and allocation of logistics resources, the low utilization rate of vehicles, and even the errors in the process of logistics delivery. Therefore, how to achieve the optimal management and allocation of logistics resources and the efficient services of routing optimization and loading of tasks is another huge challenge.

(3) Logistics industry is facing the dramatic increase in logistics demands, the environmental issues related to the logistics activities, and high logistics costs. Developing a balance among economic, environmental, and social objectives for sustainability is an urgent target for the logistics industry. Therefore, it is vital to develop a method for achieving a sustainable balance among these objectives. 

real-time information sensing model for logistics resources is built to sense and obtain real-time logistics information. The value-added logistics information is managed and shared among logistics enterprises, vehicles, and tasks. Implementing the optimal management and allocation of logistics resources is the key to maximizing the utilization of logistics resources. A new, real-time, and dynamic logistics service strategy is adopted to improve the current logistics service pattern. Real-time information enabled routing optimization and navigation, and loading services are developed to provide routing optimization and navigation for smart vehicles, and avoid incorrect loading of tasks. An IoTenabled dynamic optimization method for smart vehicles and logistics tasks (IOMVT) is developed to improve the efficiency of logistics services, and achieve green and sustainable logistics.

The rest of the paper is organized as follows. Section 2 reviews the related work. Section 3 outlines the overall architecture of IOMVT. Section 4 describes IOMVT. In Section 5, a case study is used to demonstrate the efficiency of the proposed method. Conclusions and future works are given in Section 6.

\section{Related work}

Green logistics have been ongoing for decades and taken a wide-ranging and profound reform in the logistics industry. Surrounding the projects of transportation and logistics, significant progress and research productions have been achieved in the field of logistics. Three streams of literature are relevant to this research. They are management and allocation of logistics resources, real-time information acquisition and sharing, and logistics transportation and routing optimization.

\subsection{Management and allocation of logistics resources}

Logistics management is the set of integrated logistics activities, including freight transport, storage, inventory management, materials handling, and all the related information processing that requires to move products through the efficient supply chain process (Martel and Klibi, 2016). The objective of logistics resources management is to facilitate information flow and share knowledge, and determine the most effective approach for allocating the appropriate logistics resources to different logistics functions (Ross, 2003). Green logistics management, as a novel management approach, was developed to manage logistics activities in a green and sustainable manner with environmental considerations (Lai and Wong, 2012). However, the low-efficiency logistics resources management is still a typical issue in the current logistics activities. To address this problem, many works on logistics resources management such as the use of logistics resources, the improvement of transportation service performances, and the current methods on the logistics resources management were surveyed (Crainic et al., 2004; Sheu, 2006; Poon et al., 2009). This facilitated to integrate and manage logistics resources, orders, enterprises, and customers with high efficiency, given the chaotic logistics management, especially in logistics enterprises. However, logistics resources management systems cannot sense and obtain the real-time information of logistics resources, and the data collection technique is either manual-based or barcode-based (Poon et al., 2009). The lack of the sharing of real-time information is a typical challenge faced by logistics resources management. Therefore, logistics resources management systems are incapable of implementing the real-time response, monitoring, and handling of logistics resources, especially for new tasks. The optimal allocation of logistics resources is also impossible. 

optimal allocation of logistics resources, which is quite important in green logistics. The optimal allocation of logistics resources is to maximize the utilization of logistics resources, further reduce the number of vehicles needed in the logistics activities (Liu et al., 2018). The progress of this field in research and application is significant in terms of the allocation methodologies of logistics resources (Sheu, 2006; Yang et al., 2016; Zhang et al., 2018a). It focuses on the high-quality logistics services within lower logistics costs and fewer logistics resources, therefore, provides a strategic support for current logistics practice towards green logistics. In addition, research on approaches in how to achieve the optimal allocation of logistics resources was widely developed such as vehicle assignment and order processing (Vukadinović et al., 1999; Sheu, 2006; Chow et al., 2014). Nevertheless, the decisionmaking plan of the allocation of logistics resources is carried in advance in most cases, and the frequent deviation between the execution and plan still exists because the dynamic feature of the optimal allocation of logistics resources is ignored. Therefore, it is essential to implement the optimal allocation of logistics resources driven by the real-time information which can support the real-time optimization of new tasks.

\subsection{Real-time information acquisition and sharing}

The motivation of sensing and collecting real-time information of logistics resources is to have vehicles be smart, and make dynamic optimization for smart vehicles and logistics tasks in real time. It is essential to develop real-time decision-making functions that can sense and handle exceptions throughout the whole logistics process. It can address the issues existing in current logistics decisionmaking with the lack of real-time information. Therefore, how to sense and obtain real-time information of logistics resources becomes a key issue. Research on methods, technologies, and tools in real-time information acquisition offers a good support for the collection of real-time information (Wang et al., 2011; Zhang et al., 2017a, b). Radio frequency identification (RFID) technology with high reliability and transmission speed is applied to multiple fields for collecting real-time information and has achieved remarkable results (Want, 2006). RFID can enable "process freedoms" and real-time visibility into supply chains, especially in logistics industry (Angeles, 2006). The real-time information capturing and integration framework of the Internet of Manufacturing Things (IoMT) provided a technical and strategical guidance to address the issue of sensing real-time information of logistics resources (Zhang et al., 2015). Under this framework, the configuration of IoMT sensing environment within installed RFID tags, readers, and sensors was developed to sense and capture real-time logistics information. Real-time monitoring and tracking of logistics resources can also be achieved, such as real-time monitoring of goods delivery (Ruan and Shi, 2016) and planning and execution processes (Koh and Wang, 2010). After collecting real-time information of logistics resources, instead of directly entering the optimization stage, value-added mechanisms of logistics information are entailed to achieve the processing and management of real-time information (Qu et al., 2015; Zhang et al., 2018b). Value-added information of logistics resources is transmitted in real time through the communication protocol (Sheng et al., 2013; Li et al., 2017). The sharing of value-added logistics information throughout the entire logistics process is important to real-time decision-making (Lee et al., 2000; Wang and Koh, 2010; Nativi and Lee, 2012), which can promote the optimal allocation of logistics resources in real time.

Although significant progress has been made in the above researches, there are some challenges existing in the current logistics industry, especially for the application of the real-time data-driven 

separately and mainly focused on how to apply the IoT-related techniques on the collection of real-time information. They do not take into account the profiles of the overall solution for the integration and management of whole logistics services (e.g., the configuration of smart vehicles, updating logistics information, and implementation of real-time logistics systems) or profiles of companies.

\subsection{Logistics transportation and routing optimization}

Logistics transportation is the freight transportation in the process of logistics activities, which associates with the loading of tasks and the selection of the optimal tasks, as well as tasks delivery (Zhang et al., 2016). The freight transportation constitutes a very small proportion of the total transportation time for goods, but it may represent up to $28 \%$ of the total transportation costs (MunozVillamizar et al., 2018). However, logistics transportation is in an assignment-centralized and artificial way in practice in the current logistics. Therefore, the waste of loading capacity, errors in loading tasks, and low-efficiency logistics delivery are common resulting from the lack of real-time logistics information and effective routing optimization methods (McKinnon and Edwards, 2010). Routing optimization, as the core of logistics transportation, is to improve freight delivery services in cost, distance, and energy consumption in an optimal path.

The vehicle routing problem, as one of the more widely-used logistics transportation problems, has been used to optimize the routing costs. In a recent book, Toth and Vigo (2014) systematically reviewed the research of VRP, related methods and applications. However, challenges in VRP for realtime optimization of logistics resources still exist, for example, several issues related to VRP including the real-time information of vehicles' paths, sensing logistics exceptions in real time, and delivery requests of new tasks are ignored. As a result, there is a growing attempt in recent literature in VRP to investigate the issues of real-time vehicle routing (Ghiani et al., 2003; Liao and Hu, 2011). This is because that the solutions of real-time information-driven vehicle routing can make a quick response for the logistics exceptions, and reduce the costs caused by rescheduling. Real-time tracking and locating for logistics resources could be achieved and shared among stakeholders, further, to avoid errors in the tasks delivery (Zhang et al., 2017c; Zhang et al., 2018c). In addition, real-time VRP is characterized by high dynamics and randomness of logistics tasks. This leads to the increase in the complexity of VRP. To address such challenges, a comprehensive review of real-world applications and the state-of-the-art solution methods for dynamic VRP was studied (Wang et al., 2012; Pillac et al., 2013; Okulewicz and Mańdziuk, 2017). Many algorithms of VRP focused more on the shortest delivery distance and one-way routing optimization with the consideration of cost, energy consumption, and delivery time (Yu et al., 2009; Marinakis and Marinaki, 2010; Pavone et al., 2011). This often results in the low loading rate of vehicles, the waste of logistics resources, and the increase in logistics costs. The delivery requests of new tasks cannot be sensed and handled in real time in the ongoing logistics delivery activities. As a result, the number of vehicles used in logistics delivery increases. It should be noted that the standard objective function of VRP is to minimize the total distribution distance. Most VRPs are formulated as a single objective function of minimizing the cost of the solutions (Braekers et al., 2016), despite the fact that the majority of problems in industry, particularly in logistics, are not limited to minimizing the operational costs derived from the distribution distance, and other objectives should be considered. Moreover, real-time response and handling of new tasks in VRPs is few. However, this case in the current logistics activities is quite common due to the rapid increase of logistics demands, and this issue is really worth studying. 


\section{Architecture of IoT-enabled dynamic optimization for vehicles}

\section{and tasks}

The overall architecture of IOMVT consists of three modules as shown in Fig. 1, namely smart vehicle terminals (SVT), enterprise information systems (EIS), and dynamic distribution center (DDC). The following sections will introduce the functions of each module.

EIS is used to integrate and manage logistics enterprises and resources, including enterprises' accounts, tasks, and vehicles. Logistics enterprises, especially small and medium-sized enterprises, create an account in EIS and register the information of their enterprises. It includes the information of tasks and vehicles and the individual information of each enterprise like the address, property, etc. The task management module is responsible for updating information of tasks in real time such as the current position, the destination, and the delivery date, etc. It also numbers the tasks, and documents the dimension and weight of the tasks. Then, the vehicle management module is used to integrate and manage the information of the vehicles like the vehicle's registry, the rated volume and weight, and the coding of the vehicles. Then, the tasks and vehicles are intensively managed in EIS and shared among different logistics enterprises. Finally, real-time information of vehicles and tasks can be collected and monitored in EIS. In addition, the interfaces of the logistics resources registry platform and real-time vehicle sets in EIS are introduced in Case study.

DDC is responsible for extracting vehicles' real-time information and building IOMVT based on the real-time information of logistics resources. Real-time information of vehicles and tasks is extracted and created as the vehicle sets and task sets. These information sets of vehicles and tasks contain the useful and value-added information that can be used in the optimization model after filtering. The information of task lists of the vehicles can also be created. Then, the value-added information of vehicles and tasks is transmitted to the IoT-enabled dynamic optimization module. Based on the realtime information of logistics resources, an IoT-enabled dynamic optimization method is developed to implement the optimal allocation and scheduling of logistics resources, and real-time response and optimization of new tasks. The optimal tasks can be assigned to the vehicles in a competitive manner. Further, a sustainable balance of economic, environmental, and social objectives can be achieved. Finally, high-efficiency logistics services based on optimal logistics results is developed to improve the efficiency of logistics. Real-time routing optimization and navigation services provide the optimal distribution routing for drivers and real-time sensing of exception events. Loading optimization service is designed to avoid errors in the process of logistics delivery.

SVT is used to sense and obtain the real-time information of vehicles. It is composed of three submodules. Firstly, identification devices like RFID devices and communication devices are selected and configured on the vehicles. These devices are used to make the vehicles have the capacity of sensing and obtaining the real-time information of logistics resources. Then, the real-time information of the vehicles such as the position and the task lists can be sensed and monitored. Information processing is developed to process primitive information of the vehicles to the meaningful information where an information updating mechanism of logistics resources is developed. The value-added logistics information can be shared and transmitted with other modules through the communication protocol. Finally, the visualization of the real-time information of the vehicles is developed to provide the routing optimization and navigation services. This visual interface has the ability of interactive communication 


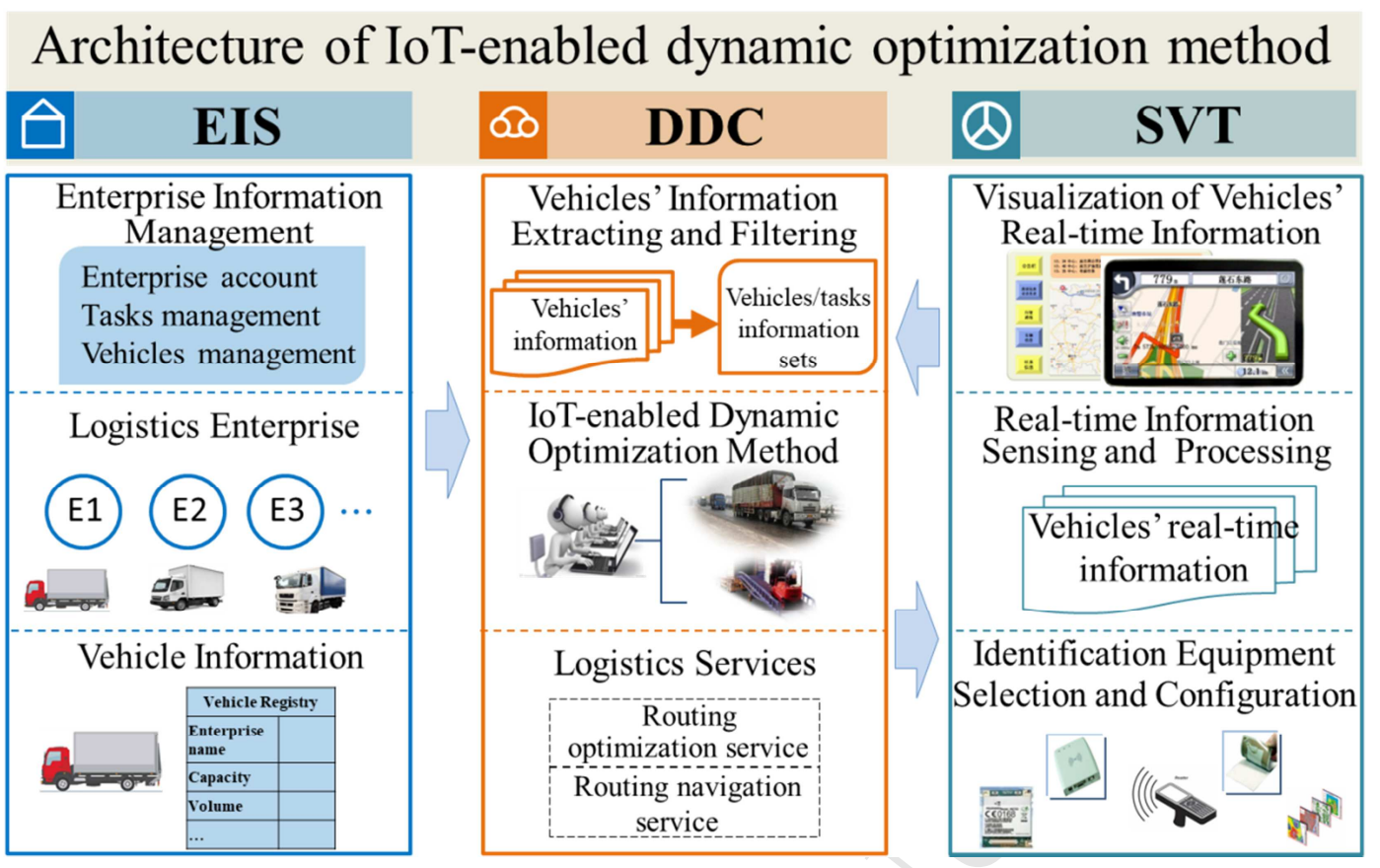

Fig. 1. The architecture of IOMVT

The overall workflow of this architecture is that the information of logistics resources registered in EIS and the real-time information of vehicles in SVT is transmitted into DDC. Then, the optimal results obtained by the IoT-enabled dynamic optimization method can be sent to the vehicles in SVT. Finally, vehicles finish the tasks assisted by the optimal logistics services. The workflow in details is introduced as follows. Firstly, logistics enterprises create an account in EIS and register their information of the enterprises, tasks, and vehicles, and EIS integrates and manages all the logistics resources registered by the logistics enterprises. Then, the information of logistics resources is transmitted to DDC that creates the vehicle sets and task sets. Then, SVT establishes direct connections with DDC, and the value-added information of vehicles and task lists on the vehicles can be timely transmitted to DDC from SVT. The optimization method for vehicles and tasks developed in DDC obtains the optimal results with a sustainable balance of economic, environmental, and social objectives. Finally, the optimal tasks assigned to the vehicles in SVT are finished based on the visualization services. The communication protocol of information transmission between DDC, EIS, and SVT is based on RFID (intranet protocol) and 4G (extranet protocol). Within the intranet protocol, logistics resources attached RFID tags can be sensed by RFID readers, and real-time information of logistics resources in EIS, DDC, and SVT can be sensed and collected. Within the extranet protocol, information transmission among EIS, DDC, and SVT is achieved.

The IoT-based real-time sensing model is the foundation of SVT and IOMVT, and includes three sub-modules as shown in Fig. 2, namely identification device selection and configuration (IDSC), realtime information sensing and processing (RISP), and information visualization (IVIS). 


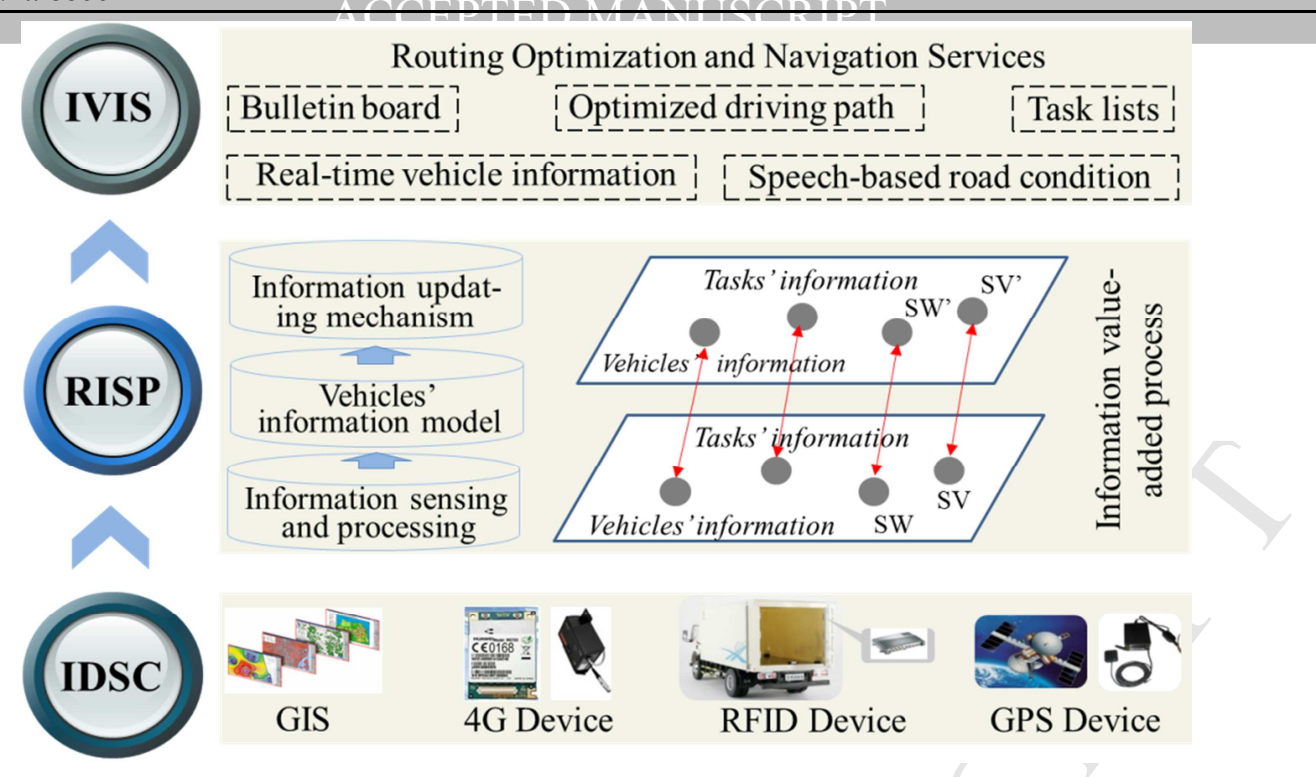

Fig. 2. The IoT-enabled real-time sensing model

Sensing devices are selected and configured into SVT to sense and transmit real-time information of vehicles and tasks in IDSC. These devices include GPS locator devices, RFID information collection devices, 4G communication devices, and integrated geographic information systems (GIS). GPS is used to locate vehicle's location; RFID information collection devices are installed on vehicle's back doors and used to collect and monitor the loading or unloading of tasks. When tasks attached RFID tags are loaded into or unloaded from vehicles, the configured RFID devices automatically collect information of logistics resources which is written in tags. This can reveal vehicles' real-time utilization rate and update the current task lists of the vehicles. Loading verification services are to ensure the consistency of information read by RFID readers and that on task lists needed to be loaded or unloaded. If tasks are loaded wrongly, the alarming will be triggered, and further to avoid errors in loading tasks. $4 \mathrm{G}$ is responsible for both-way communication between SVT and DDC. GIS is used to offer the optimal routing navigation services for drivers based on real-time task lists of vehicles.

Within the configuration of intelligent identification devices in IDSC, the real-time information sensing and processing system can sense and obtain the real-time information of the vehicles, then process and transmit the value-added real-time information to DDC. For example, when a task comes to a sensing area, this event can be sensed by the registered sensors. Then, based on the value-added information, vehicles' real-time information model is built, and includes the vehicle number, vehicle's surplus weight, surplus volume, current position, next destination, cost per kilometer, and current task lists. When receiving instructions from DDC, the sensing and processing module transfers vehicles' information to DDC in real time. The updating mechanism of real-time status information updates SW and SV once RFID readers perceive the loading/unloading of tasks (if information of the same task is read for the first time, loading information; otherwise, the unloading information). Vehicle's task lists are also updated. The updating functions are formulated as:

$$
\begin{aligned}
& \mathrm{SW}_{\mathrm{i}}^{\prime}=\mathrm{SW}_{\mathrm{i}}+\alpha^{*} \mathrm{~W}_{\mathrm{j}} \\
& \mathrm{SV}_{\mathrm{i}}^{\prime}=\mathrm{SV}_{\mathrm{i}}+\alpha^{*} \mathrm{~V}_{\mathrm{j}}
\end{aligned}
$$

Eq. (1) and Eq. (2) indicate the updating functions of the volume and weight of the vehicles. When tasks are loaded in or unloaded from the vehicles, the volume and weight of the vehicles are updated in real time. Current position, next destination, and the current task lists of the vehicles are also 
updated.

\section{Vehicle Real-time Information}

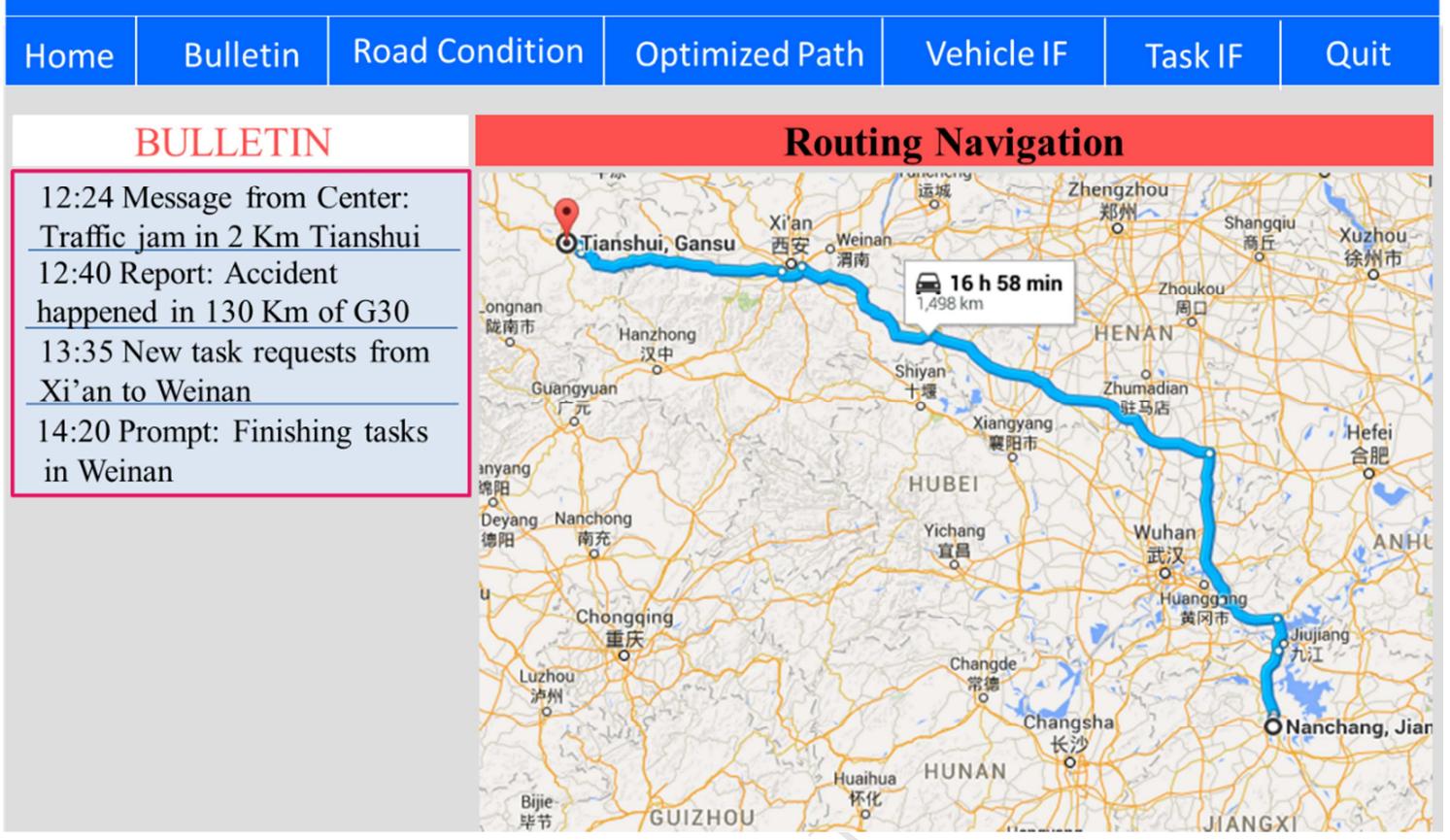

Fig. 3. The visualization of vehicles' real-time information

The real-time information sensing and processing system uploads the real-time task information of vehicles to GIS. GIS monitors current road condition and applies the path optimization software to offer the optimal routing services based on drivers' requests onto the real-time information visualization interface. Five function tabs located on the top of the visualization interface as shown in Fig. 3: Bulletin, Road Condition, Optimized Path, Vehicle IF, and Task IF, are designed to provide the real-time information of vehicles, and routing optimization and navigation services. Bulletin board on the left of the interface is used to show instructions from DDC when clicking on these tabs. For example, when clicking on Road Condition, the speech-based road information is broadcasted and shown on the bulletin board. Vehicle IF and Task IF are responsible for showing information of vehicles and tasks. Real-time routing optimization methods are applied in logistics transportation, and integrated into DDC on vehicles. In the case of unexpected events such as the traffic jam and the delivery of new tasks, the optimized routing is re-optimized and pushed to drivers if Optimized Path is clicked. The routing navigation is shown on the homepage when no other function buttons are clicked.

\section{IoT-enabled dynamic optimization method for smart vehicles and}

\section{logistics tasks}

The IoT-enabled dynamic optimization method for vehicles and tasks is developed to achieve the optimal allocation of logistics resources and real-time response and optimization for new tasks. It targets to develop a sustainable balance between economic, environmental, and social objectives for the 
current logistics activities. The whole procedure of the proposed method is shown in Fig. 4. The optimization follows 7 steps as follows:

Step 1: Initialize the vehicle sets $\mathrm{V}^{\prime}$ and task sets $\mathrm{T}^{\prime}$ based on the real-time information.

Step 2: Create the vehicle vectors $\overrightarrow{\operatorname{VID}}_{\mathrm{i}}$ and task vectors $\overrightarrow{\mathrm{TID}}_{\mathrm{j}}$ in terms of distribution information of vehicles and tasks.

Step 3: Build the pre-optimized subsets of vehicles and tasks satisfying the constraints by a circular region partition method.

Step 4: Re-optimize the task sets and vehicle sets based on the theory of the circle inscribed triangle.

Step 5: Classify the tasks according to the number of vehicles in the circular regions and their intersections.

Step 6: Optimize the tasks with multiple vehicles in the circular regions.

Step 7: Finish the tasks delivery.

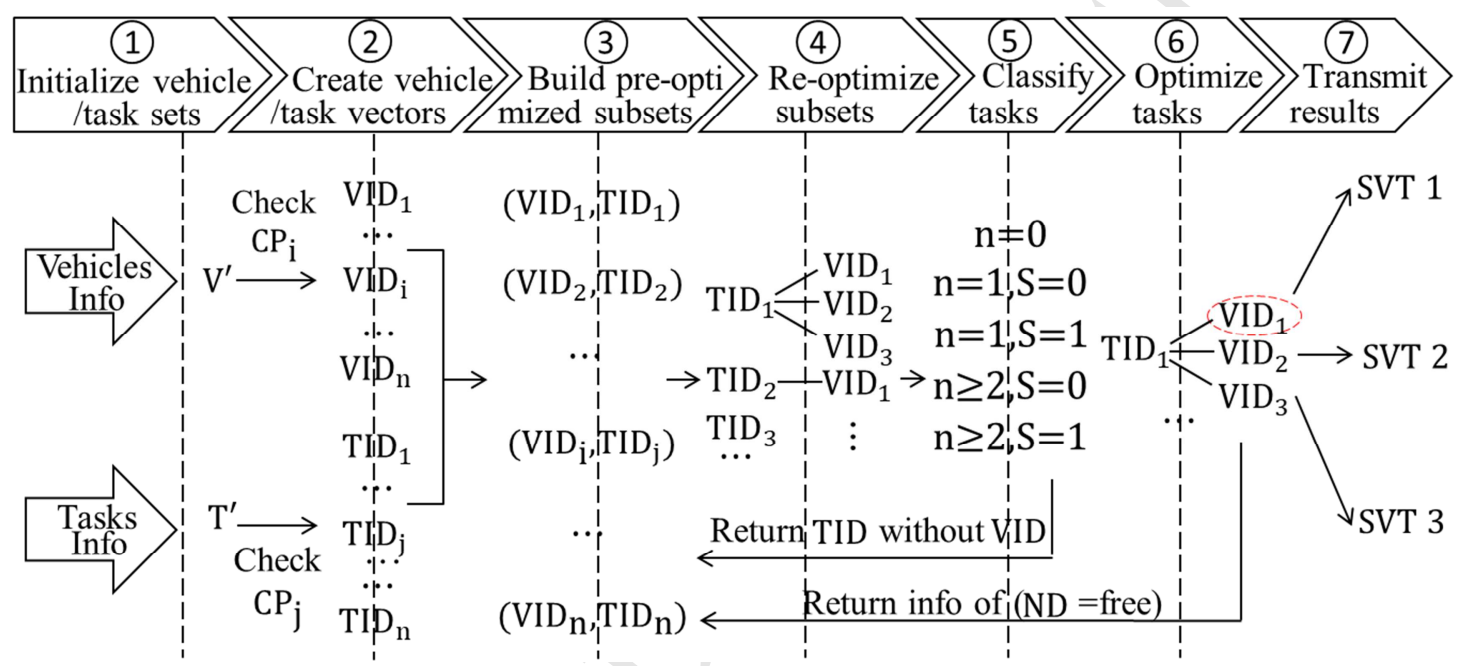

Fig. 4. The flow chart of the IOMVT

Firstly, DDC instructs SVT to transfer the real-time information of logistics resources at a certain time interval $\mathrm{t}$. $\mathrm{t}$ is a variable parameter which is inversely proportional to the scale of tasks and vehicles, and generally its default value is 5 minutes. SVT filters loading or unloading information of vehicles. Vehicle sets and task sets are created and initialized based on the real-time information of logistics resources. Vehicle sets $\mathrm{V}^{\prime}=\left\{\mathrm{VID}_{\mathrm{i}}, \mathrm{SW}_{\mathrm{i}}, \mathrm{SV}_{\mathrm{i}}, \mathrm{CP}_{\mathrm{i}}, \mathrm{ND}_{\mathrm{i}}, \mathrm{TL}_{\mathrm{i}}\right\}$, and i represents the serial number of vehicle $i$ in $V^{\prime}$. Task sets $T^{\prime}=\left\{T_{I}, W_{j}, V_{j}, C P_{j}, N D_{j}, T_{j}, d t_{j}, W_{i j}\right\}$. Vehicle vector $\overrightarrow{V I D}_{i}$ that is the distribution routing of vehicle $\mathrm{i}$ is defined as positive $\mathrm{X}$-axis $\left(\mathrm{X}^{+}\right)$. The starting and ending points of the vector are $C P_{i}$ and $\mathrm{ND}_{\mathrm{i}}$, respectively. Task vector $\overrightarrow{\mathrm{TID}_{\mathrm{j}}}$ is also created, and the starting and ending points of the vector are $C P_{j}$ and $N_{j}$, respectively. A circular region with the center of the circle $(O(R))$ located at the starting point of $\overrightarrow{\mathrm{VID}}_{\mathrm{i}}$ is established and the norm of $\overrightarrow{\mathrm{VID}}_{\mathrm{i}}$ is the radius of the circle (R). Vehicle vector $\overrightarrow{\mathrm{VID}}_{\mathrm{i}}$, task vector $\overrightarrow{\mathrm{TID}}_{\mathrm{j}}$, and $\mathrm{R}$ are formulated as follows:

$$
\begin{gathered}
\overrightarrow{\mathrm{VID}}_{\mathrm{i}}=\overrightarrow{\mathrm{CP}_{\mathrm{i}} \mathrm{ND}_{\mathrm{i}}}=\left(\mathrm{CP}_{\mathrm{i}}, \mathrm{ND}_{\mathrm{i}}\right)=\left[\left(\mathrm{x}_{\mathrm{vi}}, \mathrm{y}_{\mathrm{vi}}\right) \rightarrow\left(\mathrm{x}_{\mathrm{vi}}^{\prime}, \mathrm{y}_{\mathrm{vi}}^{\prime}\right)\right] \\
\overrightarrow{\mathrm{TID}_{\mathrm{j}}}=\overrightarrow{\mathrm{CP}_{\mathrm{j}} \mathrm{ND}_{\mathrm{j}}}=\left(\mathrm{CP}_{\mathrm{j}}, \mathrm{ND}_{\mathrm{j}}\right)=\left[\left(\mathrm{x}_{\mathrm{tj}}, \mathrm{y}_{\mathrm{tj}}\right) \rightarrow\left(\mathrm{x}_{\mathrm{tj}}^{\prime}, \mathrm{y}_{\mathrm{tj}}^{\prime}\right)\right]
\end{gathered}
$$




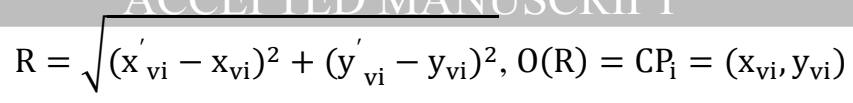

The number of tasks is huge, while that of vehicles is limited. Therefore, the vehicles in the circular regions select tasks meeting constraints, and this is defined as the circular region partition method that can effectively decrease the complexity of optimization for tasks and vehicles. The vehicles in the circular regions actively select the optimal tasks in a competitive way once delivery requests of new tasks are released. The circular region partition method is used to build the pre-optimal subsets of the vehicles and tasks. A task is defined as a point that indicates the starting point of the task. A circular region contains $n(n \geq 0)$ tasks. It is an assumption that each vehicle in the circular regions is an independent element. If the tasks locate at the intersection of the circular regions, $S=1$; otherwise, $S=0$ (intersection: $S=1$ and no-intersection: $S=0$ ). If $n=0$, then this circular region is released and the planned distribution routing is executed. If $n=1, S=0$, the tasks are directly assigned to the vehicles. If $n=1, S=1$, the tasks are assigned to the vehicles within the circular region with the minimal $R$. If $n \geq 2, S=0$, the tasks with the minimal total distance are assigned to the vehicles. If $\mathrm{n} \geq 2, \mathrm{~S}=1$, the tasks with the minimal total distance are assigned to the vehicles within the circular region with the minimal $\mathrm{R} . \overrightarrow{\mathrm{TID}}_{\mathrm{j}}=\mathrm{k}\left(\overrightarrow{\mathrm{VID}}_{\mathrm{i}}\right), \mathrm{k} \neq 0 . \mathrm{k}>0$ means that $\overrightarrow{\mathrm{TID}}_{\mathrm{j}}$ points to $\overrightarrow{\mathrm{VID}}_{\mathrm{i}}$ (positive $X$-axis: $X^{+}$); otherwise, $\overrightarrow{T I D}_{j}$ points to the negative $X$-axis $\left(X^{-}\right)$. If $n \neq 0, k \neq 0$. Here, the loading constraints for tasks are formulated as follows.

$$
\begin{gathered}
I_{1}=\left\{\begin{array}{c}
0,(\mathrm{lt} \leq \mathrm{lv}) \\
1,(\text { otherwise })
\end{array}, I_{2}=\left\{\begin{array}{c}
0,(\text { wt } \leq \mathrm{wv}) \\
1,(\text { otherwise })
\end{array},\right.\right. \\
\mathrm{I}_{3}=\left\{\begin{array}{c}
0,(\text { ht } \leq \mathrm{hv}) \\
1,(\text { otherwise) }
\end{array}, I_{4}=\left\{\begin{array}{l}
0,\left(\mathrm{~W}_{\mathrm{j}} \leq \mathrm{SW}_{\mathrm{i}}^{\prime}\right) \\
1,(\text { otherwise) }
\end{array}\right.\right. \\
\mathrm{I}=\mathrm{I}_{1}+\mathrm{I}_{2}+\mathrm{I}_{3}+\mathrm{I}_{4}
\end{gathered}
$$

Variable I is a multi-objective constraint function. $\mathrm{I}=0$ indicates that tasks can be loaded on the vehicle. Otherwise, rejecting tasks. For simplicity, the gap between tasks when loading is neglected in this paper. Tasks are reclassified and re-optimized after filtered by constraints. For the elements in the intersection regions, the element in the region with the minimal $\mathrm{R}$ is selected as a final result. This is based on the theory of the circle inscribed triangle. Besides, the elements in the no-intersection regions do not change.

If $\mathrm{n} \geq 2$, the theory of the circle inscribed triangle is used to re-optimize the task and vehicle sets, and the tasks that meet all the following conditions are selected.

Condition 1: the angle between $\operatorname{VID}_{\mathrm{i}}$ and $\mathrm{TID}_{\mathrm{j}}$ is the minimum. The equation is formulated as follows:

$$
f\left(\operatorname{VID}_{\mathrm{i}}\right)=\min _{\mathrm{j}=1}^{\mathrm{n}} \arccos \frac{\operatorname{VID}_{\mathrm{i}} * \operatorname{TID}_{\mathrm{j}}}{\left|\operatorname{VID}_{\mathrm{i}}\right| *\left|\mathrm{TID}_{\mathrm{j}}\right|}, \mathrm{i}, \mathrm{j} \in(1, \mathrm{n})
$$

Condition 2: the distance from the midpoint of $\left|\overrightarrow{\mathrm{CP}_{\mathrm{i}} N \mathrm{D}_{\mathrm{i}}}\right|$ to the midpoint of $\left|\overrightarrow{\mathrm{CP}_{\mathrm{j}} \mathrm{ND}_{\mathrm{j}}}\right|$ is the shortest. The formula is shown as below:

$$
\mathrm{d}=\min _{\mathrm{vi}, \mathrm{tj} \in(1, \mathrm{n})}\left(\left(\frac{\mathrm{x}_{\mathrm{vi}}+\mathrm{x}^{\prime}{ }_{\mathrm{vi}}}{2}-\frac{\mathrm{x}_{\mathrm{tj}}+\mathrm{x}^{\prime}{ }_{\mathrm{tj}}}{2}\right)^{2}+\left(\frac{\mathrm{y}_{\mathrm{vi}}+\mathrm{y}^{\prime}{ }_{\mathrm{vi}}}{2}-\frac{\mathrm{y}_{\mathrm{tj}}+\mathrm{y}^{\prime}{ }_{\mathrm{tj}}}{2}\right)^{2}\right), \mathrm{i}, \mathrm{j} \in(1, \mathrm{n})
$$

Condition 3: the distance of $\operatorname{VID}_{\mathrm{i}}$ finishing $\mathrm{TID}_{\mathrm{j}}$ is minimal.

$$
\mathrm{D}=\min \left[\left|\overrightarrow{\mathrm{VID}}_{\mathrm{i}}\right|+\left|\overrightarrow{\mathrm{TID}}_{\mathrm{j}}\right|+\left|{\overrightarrow{\mathrm{CP}} \mathrm{i} \mathrm{P}_{\mathrm{j}}}\right|+\mid{\overrightarrow{\mathrm{ND}_{\mathrm{i}} \mathrm{ND}_{\mathrm{j}}}}_{\mid}\right]
$$

$\mathrm{TID}_{\mathrm{j}}$ is classified into four kinds of tasks based on information of positions. (1) $\mathrm{CP}_{\mathrm{j}}, \mathrm{ND}_{\mathrm{j}} \in \mathrm{O}(\mathrm{R}) \wedge$ $\mathrm{X}^{+}$; (2) $\mathrm{CP}_{\mathrm{j}}, \mathrm{ND}_{\mathrm{j}} \in \mathrm{X}^{+} ; \mathrm{ND}_{\mathrm{j}} \notin \mathrm{O}(\mathrm{R}) \ni \mathrm{CP}_{\mathrm{j}} ;$ (3) $\mathrm{CP}_{\mathrm{j}} \notin \mathrm{X}^{+} \ni \mathrm{ND}_{\mathrm{j}} ; \mathrm{CP}_{\mathrm{j}} \notin \mathrm{O}(\mathrm{R}) \ni \mathrm{ND}_{\mathrm{j}} ;$ (4) $\mathrm{CP}_{\mathrm{j}}, \mathrm{ND}_{\mathrm{j}} \notin$ 

circular region and the positive $\mathrm{X}$-axis. Class 2 indicates that the current position of task $\mathrm{j}$ locates in the circular region and the positive $\mathrm{X}$-axis, while the destination is outside. Class 3 indicates that the destination of task $\mathrm{j}$ locates in the circular region and the positive $\mathrm{X}$-axis, while the current position is outside. Class 4 indicates that the current position and destination of task j locate outside of the circular region. Tasks that are not allocated to the vehicles return to $\mathrm{T}^{\prime}$ and are re-optimized until all the tasks are completed.

The objective function is formulated as follows, and it is composed of the total cost of tasks delivery and the cost of delay penalty.

$$
\begin{gathered}
\mathrm{C}=\min \operatorname{cost}\left(\mathrm{C}_{1}+\mathrm{C}_{3}\right) \\
\mathrm{C}_{1}=\sum_{\mathrm{i}} \sum_{\mathrm{j}} \mathrm{VC}_{\mathrm{i}} \mathrm{L}_{\mathrm{ij}}=\sum_{\mathrm{i}} \mathrm{VC}_{\mathrm{i}} \mathrm{L}_{\mathrm{i}} \\
\mathrm{C}_{2}=\sum_{\mathrm{i}} \mathrm{VC}_{\mathrm{i}} \mathrm{L}_{\mathrm{i}}^{\prime} \\
\mathrm{C}_{3}=\sum_{\mathrm{i}} \sum_{\mathrm{j}} \mathrm{TID}_{\mathrm{j}} \mathrm{w}_{\mathrm{ij}} \mathrm{T}_{\mathrm{j}} \\
\mathrm{T}_{\mathrm{j}}=\left\{\begin{array}{c}
0, \mathrm{t}_{\mathrm{j}}<\mathrm{dt}_{\mathrm{j}} \\
\mathrm{t}_{\mathrm{j}}, \mathrm{t}_{\mathrm{j}} \geq \mathrm{dt}_{\mathrm{j}}, \mathrm{t}_{\mathrm{j}}=\left(\mathrm{L}_{\mathrm{j}}-\mathrm{L}_{\mathrm{j}}^{\prime}\right) / \mathrm{L}_{\mathrm{j}}^{\prime} \\
\mathrm{C}_{0}=\sum_{\mathrm{i}} \sum_{\mathrm{j}} \mathrm{VC}_{\mathrm{i}} \mathrm{L}_{\mathrm{j}}^{\prime}
\end{array}\right.
\end{gathered}
$$

where Eq. (10) denotes that the total logistics cost of delivering all the tasks. Eq. (11) describes the delivery cost of vehicles completing all the tasks, including the accepted task $\mathrm{TID}_{\mathrm{j}}$. Eq. (12) indicates that the cost of vehicles accomplishing the tasks on the task lists, and $\mathrm{L}_{\mathrm{i}}$ is the total distance of vehicles finishing the tasks on the task lists. Eq. (13) indicates the delayed delivery penalty cost caused by accepting $\operatorname{TID}_{\mathrm{j}}$. Eq. (14) denotes a piecewise linear function, where $t_{j}$ and $d t_{j}$ are the time of delay and the allowed time of delay of $\mathrm{TID}_{\mathrm{j}}$ on the task lists. Eq. (15) indicates the cost of finishing all the tasks in the centralized and one-way logistics service.

Tasks are assigned to vehicles that have the minimum value of the objective function. The optimal distribution results are obtained and transmitted to SVT in real time. To demonstrate the efficiency of the proposed method, evaluation functions are formulated as follows:

$$
\begin{gathered}
\mathrm{V}^{\prime}=\sum_{\mathrm{i}}\left(\overline{\mathrm{V}}_{\mathrm{i}} \overline{\mathrm{L}}_{\mathrm{i}}\right) /\left(\mathrm{V}_{\mathrm{i}} \mathrm{L}_{\mathrm{i}}\right)=\sum_{\mathrm{j}} \mathrm{V}_{\mathrm{j}} \mathrm{L}_{\mathrm{j}} / \sum_{\mathrm{i}} \mathrm{V}_{\mathrm{i}} \mathrm{L}_{\mathrm{i}} \\
\mathrm{W}^{\prime}=\sum_{\mathrm{i}}\left(\overline{\mathrm{W}}_{\mathrm{i}} \overline{\mathrm{L}}_{\mathrm{i}}\right) /\left(\mathrm{W}_{\mathrm{i}} \mathrm{L}_{\mathrm{i}}\right)=\sum_{\mathrm{j}} \mathrm{W}_{\mathrm{j}} \mathrm{L}_{\mathrm{j}} / \sum_{\mathrm{i}} \mathrm{W}_{\mathrm{i}} \mathrm{L}_{\mathrm{i}} \\
\mathrm{R}^{\prime}=\left(\mathrm{V}^{\prime}, \mathrm{W}^{\prime}\right) \\
\mathrm{TFC}=\sum_{\mathrm{i}} \mathrm{L}_{\mathrm{i}} \mathrm{FC}_{\mathrm{i}} \\
\overline{\mathrm{FC}}=\mathrm{TFC} / \sum_{\mathrm{i}} \mathrm{L}_{\mathrm{i}} \\
\mathrm{C}^{\prime}=\left[\mathrm{C}-\mathrm{C}_{0}\right] / \mathrm{C}_{0} \\
\overline{\mathrm{R}}=[\mathrm{N}-\overline{\mathrm{N}}] / \mathrm{N}
\end{gathered}
$$

Eq. (16) and (17) represent the average effective loading rate of vehicles (V') and the average effective weight of vehicles (W'). Eq. (16) indicates that the product of the real-time loading volume of vehicles and the distance of vehicles in real time divides the product of the volume of vehicles and the total distance of finishing tasks. Eq. (17) denotes that the product of the real-time weight of vehicles and the distance of vehicles in real time divides the product of the total weight of vehicles and the total distance of finishing tasks. Eq. (18) indicates the average loading rate of vehicles (R), which reflects the average effective loading rate and weight of vehicles as a whole. Eq. (19) and (20) represent the total fuel consumption, and the average fuel consumption per kilometer. Eq. (21) and (22) indicate the rate of saving cost and the utilization rate of vehicles. 


\section{Case study}

\subsection{A case study for optimization of tasks and vehicles}

A case study is used to demonstrate the efficiency of the proposed IoT-enabled dynamic optimization method for tasks and vehicles. Before conducting the case study, logistics resources registry and integration in EIS discussed in Section 3 is introduced as shown in Fig. 5 where logistics enterprise registry platform and real-time vehicle sets are presented. Firstly, logistics enterprises register and login the logistics resources registry platform, and then choose the right user roles and enter the interface of this platform. Logistics enterprises can edit and submit their enterprise information and vehicle information on this platform. The enterprise information includes enterprise name, number, and address. The vehicle information consists of vehicle name, number, length, width, height, current weight, and the maximum weight, etc. Finally, the integration of vehicles and tasks from different enterprises will be accomplished. Real-time vehicle sets and task sets can be established. When clicking on Vehicle Set as shown in the bottom left of Fig. 5, real-time information of vehicles can be shown. Vehicle $1\left(\mathrm{VID}_{1}\right)$ is taken as an example to introduce the real-time information of vehicles. It includes the current position, the next destination, surplus weight and volume, the task lists on vehicles, and quality of services. The information of tasks on the task lists of vehicles can also be shown. Tables 1 and 2 show the information of vehicles and tasks at time T. Table 3 is the task list information of the vehicles. 


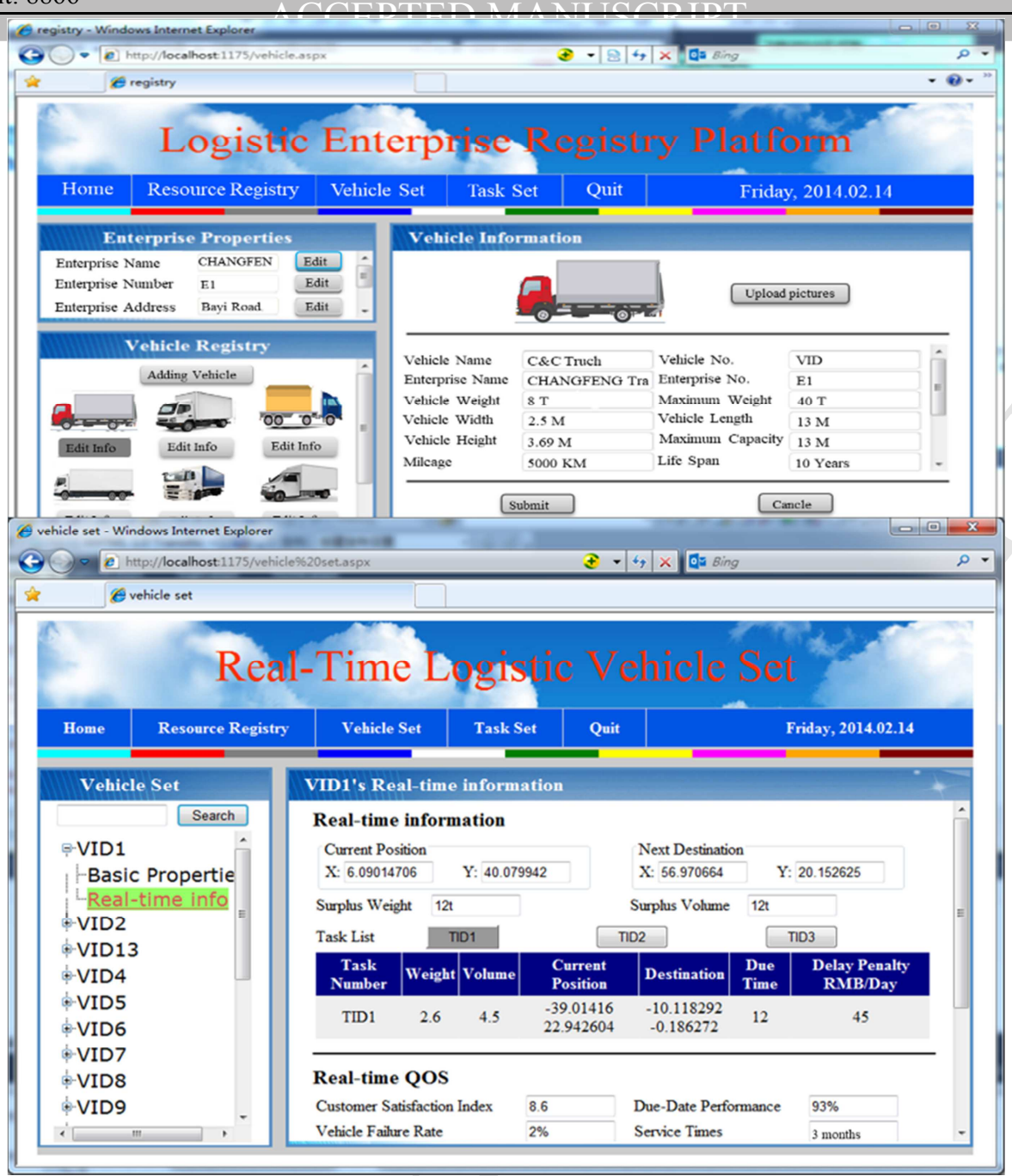

Fig. 5. Logistics resources registry and integration

Table 1 Vehicle information at time $\mathrm{T}$

\begin{tabular}{|c|c|c|c|c|c|c|c|c|c|}
\hline \multirow{2}{*}{ VID } & \multirow{2}{*}{$\mathrm{SW} / \mathrm{t}$} & \multirow{2}{*}{$\mathrm{SV} / \mathrm{m}^{3}$} & \multicolumn{2}{|c|}{$\mathrm{CP}$} & \multicolumn{2}{|c|}{ ND } & \multirow{2}{*}{$\begin{array}{c}\mathrm{VC} \\
(\$ / \mathrm{Km})\end{array}$} & \multirow{2}{*}{$\begin{array}{c}\text { OC } \\
(\mathrm{L} / \mathrm{Km})\end{array}$} & \multirow{2}{*}{$\mathrm{TL}$} \\
\hline & & & $\mathrm{X}(\mathrm{Km})$ & $\mathrm{Y}(\mathrm{Km})$ & $\mathrm{X}(\mathrm{Km})$ & $\mathrm{Y}(\mathrm{Km})$ & & & \\
\hline $\mathrm{VID}_{1}$ & 12 & 17 & 6.1 & 40.1 & 56.9 & 20.2 & 1.6 & 0.24 & $\mathrm{TL}_{1}$ \\
\hline $\mathrm{VID}_{2}$ & 13 & 15 & 33.1 & -10.7 & 5.9 & -18.8 & 1.6 & 0.24 & $\mathrm{TL}_{2}$ \\
\hline $\mathrm{VID}_{3}$ & 14 & 23 & 55.4 & 68.4 & 70.1 & 2.1 & 1.6 & 0.24 & $\mathrm{TL}_{3}$ \\
\hline $\mathrm{VID}_{4}$ & 5.4 & 8.1 & -28.1 & 33.5 & -14.0 & 14.5 & 1.6 & 0.24 & $\mathrm{TL}_{4}$ \\
\hline $\mathrm{VID}_{5}$ & 7.5 & & -31.7 & 20.9 & -5.1 & 1.3 & 1.4 & 0.24 & $\mathrm{TL}_{5}$ \\
\hline $\mathrm{VID}_{6}$ & 12 & 16 & -29.8 & -32.4 & 45.6 & -12.8 & 1.4 & 0.24 & $\mathrm{TL}_{6}$ \\
\hline $\mathrm{VID}_{7}$ & 16.8 & 19 & -27.1 & -49.4 & -77.3 & -21.3 & 1.8 & 0.24 & $\mathrm{TL}_{7}$ \\
\hline $\mathrm{VID}_{8}$ & 9 & 12 & -97.9 & 1.1 & -8.1 & 18.7 & 2.1 & 0.24 & $\mathrm{TL}_{8}$ \\
\hline $\mathrm{VID}_{9}$ & 17 & 21 & -19.5 & -75.8 & -38.6 & -70.3 & 2.1 & 0.32 & $\mathrm{TL}_{9}$ \\
\hline $\mathrm{VID}_{10}$ & 16 & 13 & -37.8 & -12.9 & 2.5 & -37.1 & 2.1 & 0.32 & $\mathrm{TL}_{10}$ \\
\hline $\mathrm{VID}_{11}$ & 16 & 16 & 8.8 & -49.1 & -38.3 & -56.9 & 2.1 & 0.32 & $\mathrm{TL}_{11}$ \\
\hline $\mathrm{VID}_{12}$ & 27 & 29 & 31.3 & -83.8 & 9.5 & -31.0 & 2.1 & 0.32 & $\mathrm{TL}_{12}$ \\
\hline $\mathrm{VID}_{13}$ & 26 & 37 & 36.9 & -56.6 & 80.4 & -7.5 & 2.6 & 0.32 & $\mathrm{TL}_{13}$ \\
\hline $\mathrm{VID}_{14}$ & 15 & 31 & 43.9 & 10.8 & 81.8 & 21.8 & 2.6 & 0.32 & $\mathrm{TL}_{14}$ \\
\hline $\mathrm{VID}_{15}$ & 16 & 23 & 21.1 & -22.8 & 28.5 & -35.4 & 2.6 & 0.4 & $\mathrm{TL}_{15}$ \\
\hline $\mathrm{VID}_{16}$ & 21 & 27 & -25.8 & 68.70 & 52.5 & 33.7 & 2.6 & 0.4 & $\mathrm{TL}_{16}$ \\
\hline $\mathrm{VID}_{17}$ & 10 & 21 & 31.5 & -26.1 & 41.2 & -53.4 & 2.6 & 0.4 & $\mathrm{TL}_{17}$ \\
\hline $\mathrm{VID}_{18}$ & 16 & 32 & 5.8 & 70.8 & 60.2 & -8.7 & 2.6 & 0.4 & $\mathrm{TL}_{18}$ \\
\hline $\mathrm{VID}_{19}$ & 17 & 24 & 0.8 & 68.67 & 71.2 & -5.2 & 2.6 & 0.4 & $\mathrm{TL}_{19}$ \\
\hline
\end{tabular}


Table 2 Task information at time $\mathrm{T}$

\begin{tabular}{|c|c|c|c|c|c|c|}
\hline \multirow{2}{*}{$\begin{array}{l}\text { Task } \\
\text { No. }\end{array}$} & \multirow{2}{*}{$\mathrm{W} / \mathrm{t}$} & \multirow{2}{*}{$\mathrm{V} / \mathrm{m}^{3}$} & \multicolumn{2}{|c|}{$\mathrm{CP}$} & \multicolumn{2}{|c|}{ ND } \\
\hline & & & $\mathrm{X}(\mathrm{Km})$ & $\mathrm{Y}(\mathrm{Km})$ & $\mathrm{X}(\mathrm{Km})$ & $\mathrm{Y}(\mathrm{Km})$ \\
\hline $\mathrm{TID}_{1}$ & 2.6 & 4.5 & -39.0 & 22.9 & -10.1 & -0.2 \\
\hline $\mathrm{TID}_{2}$ & 6.2 & 14 & 35.3 & 12.5 & 64.9 & 19.4 \\
\hline $\mathrm{TID}_{3}$ & 6.3 & 7 & 15.8 & -88.0 & 0.6 & -20.1 \\
\hline $\mathrm{TID}_{4}$ & 8.2 & 13 & 21.4 & -57.2 & -18.9 & -36.1 \\
\hline $\mathrm{TID}_{5}$ & 7.4 & 7.1 & -80.1 & 1.7 & -18.0 & 33.1 \\
\hline $\mathrm{TID}_{6}$ & 4.5 & 9 & -34.4 & -17.9 & 20.4 & -34.9 \\
\hline $\mathrm{TID}_{7}$ & 10 & 15 & 33.5 & -18.5 & 64.9 & -43.7 \\
\hline $\mathrm{TID}_{8}$ & 7 & 13 & 55.2 & 39.4 & 62.4 & -3.3 \\
\hline $\mathrm{TID}_{9}$ & 14 & 18 & 56.2 & -51.6 & 81.4 & -17.6 \\
\hline $\mathrm{TID}_{10}$ & 4.8 & 6.3 & -19.2 & -57.5 & -72.7 & -23.2 \\
\hline $\mathrm{TID}_{11}$ & 2.6 & 9.4 & 10.1 & 42.1 & 50.3 & 21.3 \\
\hline $\mathrm{TID}_{12}$ & 4.2 & 6.9 & 43.2 & -54 & -50.8 & -56 \\
\hline $\mathrm{TID}_{13}$ & 1.3 & 1.3 & -20.2 & 16.3 & -9.6 & 4.9 \\
\hline $\mathrm{TID}_{14}$ & 3.2 & 5.5 & -44.3 & -5.6 & -10.6 & 15.4 \\
\hline $\mathrm{TID}_{15}$ & 5.5 & 3 & 36.2 & 31.3 & 60.2 & 20.3 \\
\hline $\mathrm{TID}_{16}$ & 6.4 & 3.6 & -20.8 & -22.2 & -4.5 & -23.4 \\
\hline $\mathrm{TID}_{17}$ & 2.3 & 7.3 & 32.5 & -50 & 59.3 & -30.7 \\
\hline TID $_{18}$ & 4.4 & 2.3 & 20.2 & -17.3 & -6.2 & -19.9 \\
\hline TID $_{19}$ & 2 & 3.6 & 57.4 & 13.2 & 68.3 & 4.7 \\
\hline $\mathrm{TID}_{20}$ & 3.7 & 4.5 & 20.70 & 32.1 & 48.2 & 19.1 \\
\hline $\mathrm{TID}_{21}$ & 3 & 2.1 & 69.3 & 3.7 & 74.2 & 1.5 \\
\hline $\mathrm{TID}_{22}$ & 9.4 & 8 & -26.7 & -64 & -36.1 & -64.2 \\
\hline $\mathrm{TID}_{23}$ & 2 & 3 & -26.2 & 29.3 & -16.0 & 17.2 \\
\hline $\mathrm{TID}_{24}$ & 1 & 2 & 21.1 & 23.9 & -12.1 & 12 \\
\hline $\mathrm{TID}_{25}$ & 1.1 & 2 & -15.7 & 12.9 & -1.5 & -4.4 \\
\hline $\mathrm{TID}_{26}$ & 3.7 & 8.5 & 30.2 & -11.4 & 18.2 & -16.3 \\
\hline $\mathrm{TID}_{27}$ & 2.7 & 7.4 & 7.9 & -26.6 & 40 & -18.1 \\
\hline $\mathrm{TID}_{28}$ & 2 & 4.2 & 60.2 & 48.3 & 70.2 & 3.8 \\
\hline $\mathrm{TID}_{29}$ & 2.1 & 6.8 & 58.2 & 0 & 65 & -11.2 \\
\hline $\mathrm{TID}_{30}$ & 4.3 & 3.9 & -23.2 & -46.2 & -47.6 & -33.7 \\
\hline $\mathrm{TID}_{31}$ & 2.5 & 4 & -60 & -40 & -73 & -17.4 \\
\hline $\mathrm{TID}_{32}$ & 8.1 & 4.5 & -80 & -19.2 & -81 & -34.9 \\
\hline $\mathrm{TID}_{33}$ & 4.3 & 3.2 & -92 & -10 & -49.7 & 14 \\
\hline $\mathrm{TID}_{34}$ & 16.8 & 19.9 & -15.3 & -8.5 & -30.1 & -65.4 \\
\hline $\mathrm{TID}_{35}$ & 4.9 & 6.9 & 57.6 & -40.3 & 79.4 & -5.7 \\
\hline $\mathrm{TID}_{36}$ & 10.9 & 6.3 & 70.3 & 0 & 86.7 & -17.3 \\
\hline $\mathrm{TID}_{37}$ & 3.2 & 1.3 & -35.2 & -20 & -20.3 & -15.2 \\
\hline TID $_{38}$ & 2.5 & 4.5 & 47.8 & -13.2 & 50.8 & -1 \\
\hline $\mathrm{TID}_{39}$ & 6.9 & 2.4 & -6 & -37.2 & -6 & -30 \\
\hline $\mathrm{TID}_{40}$ & 9.3 & 19 & 33.2 & 40.7 & 50.1 & 6 \\
\hline TID $_{41}$ & 7.9 & 5.6 & 22.3 & -57.3 & 8.1 & -28.5 \\
\hline $\mathrm{TID}_{42}$ & 7.2 & 10 & -23.8 & 75 & 0 & 50.3 \\
\hline $\mathrm{TID}_{43}$ & 5.1 & 6.9 & 30.6 & 60.2 & 50.1 & 30.1 \\
\hline $\mathrm{TID}_{44}$ & 4.7 & 17.3 & 10 & 80.1 & 35.7 & 60.3 \\
\hline $\mathrm{TID}_{45}$ & 2.4 & 3.1 & -14 & 14.5 & -11.4 & 10.9 \\
\hline $\mathrm{TID}_{46}$ & 2.2 & 1.4 & -32.2 & 24.7 & 12.3 & 4.4 \\
\hline $\mathrm{TID}_{47}$ & 2 & 7.6 & 32.8 & -24.3 & 35.2 & -37.2 \\
\hline $\mathrm{TID}_{48}$ & 6.3 & 8 & 0 & -60.2 & -22.3 & -53.4 \\
\hline TID $_{49}$ & 4.3 & 3.1 & 28 & -82.1 & 20 & -60.5 \\
\hline TID $_{50}$ & 3 & 4.8 & 36.3 & -40 & 40.9 & -54.8 \\
\hline TID $_{51}$ & 3.7 & 5.1 & 7.0 & 76.5 & 25 & 49.6 \\
\hline TID $_{52}$ & 8.1 & 12 & 50.1 & 13.6 & 75.5 & 19.5 \\
\hline TID $_{53}$ & 4.5 & 4.1 & 26.4 & -14.1 & 6.2 & -17.6 \\
\hline
\end{tabular}




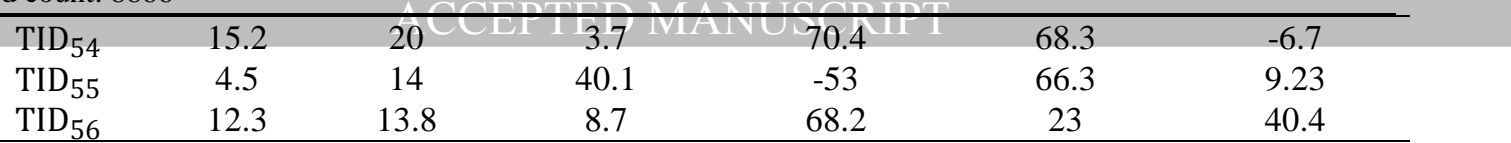

Table 3 Task list information of vehicles

\begin{tabular}{|c|c|c|c|c|c|c|c|c|c|}
\hline \multirow{2}{*}{$\begin{array}{l}\text { TL } \\
\text { No. }\end{array}$} & \multirow{2}{*}{$\begin{array}{l}\text { Task } \\
\text { No. }\end{array}$} & \multirow{2}{*}{$\mathrm{W} / \mathrm{t}$} & \multirow{2}{*}{$\mathrm{V} / \mathrm{m}^{3}$} & \multicolumn{2}{|c|}{ CP } & \multicolumn{2}{|c|}{ ND } & \multirow{2}{*}{$\mathrm{dt}_{\mathrm{j}} / \mathrm{day}$} & \multirow{2}{*}{$\mathrm{w}_{\mathrm{ij}} / \$$} \\
\hline & & & & $\mathrm{X}(\mathrm{Km})$ & $\mathrm{Y}(\mathrm{Km})$ & $\mathrm{X}(\mathrm{Km})$ & $\mathrm{Y}(\mathrm{Km})$ & & \\
\hline \multirow{2}{*}{$\mathrm{TL}_{1}$} & $\mathrm{TID}_{1}$ & 9 & 3.6 & 6.1 & 40.1 & 56.9 & 20.2 & 0.5 & 60 \\
\hline & $\mathrm{TID}_{2}$ & 6 & 4.5 & 6.1 & 40.1 & 60.9 & 23.5 & 1 & 80 \\
\hline $\mathrm{TL}_{2}$ & $\mathrm{TID}_{1}$ & 7 & 2.3 & 33.1 & -10.7 & 5.9 & -18.8 & 0.46 & 45 \\
\hline $\mathrm{TL}_{3}$ & $\mathrm{TID}_{1}$ & 6 & 6.1 & 55.4 & 68.4 & 70.1 & 2.1 & 0.6 & 65 \\
\hline $\mathrm{TL}_{4}$ & $\mathrm{TID}_{1}$ & 10 & 1.3 & -28.1 & 33.5 & -14.0 & 14.5 & 0.7 & 56 \\
\hline $\mathrm{TL}_{5}$ & $\mathrm{TID}_{1}$ & 7.5 & 6 & -31.7 & 20.9 & -5.1 & 1.3 & 0.8 & 42 \\
\hline $\mathrm{TL}_{6}$ & $\mathrm{TID}_{1}$ & 10 & 8.3 & -29.8 & -32.4 & 45.6 & -12.8 & 0.4 & 35 \\
\hline $\mathrm{TL}_{7}$ & $\mathrm{TID}_{1}$ & 12 & 5.6 & -27.1 & -49.4 & -77.3 & -21.3 & 1 & 27 \\
\hline $\mathrm{TL}_{9}$ & $\mathrm{TID}_{1}$ & 8 & 7.2 & -19.5 & -75.8 & -38.6 & -70.3 & 1.2 & 34 \\
\hline $\mathrm{TL}_{10}$ & $\mathrm{TID}_{1}$ & 6 & 3.2 & -37.8 & -12.9 & 2.5 & -37.1 & 1.1 & 54 \\
\hline \multirow{2}{*}{$\mathrm{TL}_{11}$} & $\mathrm{TID}_{1}$ & 8 & 4.5 & 8.8 & -49.1 & -38.3 & -56.9 & 0.7 & 25 \\
\hline & $\mathrm{TID}_{2}$ & 6 & 25 & 8.8 & -49.1 & -48.9 & -32.8 & 0.8 & 47 \\
\hline $\mathrm{TL}_{13}$ & $\mathrm{TID}_{1}$ & 4 & 3.5 & 36.9 & -56.6 & 80.4 & -7.5 & 0.9 & 35 \\
\hline $\mathrm{TL}_{14}$ & $\mathrm{TID}_{1}$ & 7 & 2.9 & 43.9 & 10.8 & 81.8 & 21.8 & 0.8 & 41 \\
\hline $\mathrm{TL}_{15}$ & $\mathrm{TID}_{1}$ & 8 & 7.2 & 21.1 & -22.8 & 28.5 & -35.4 & 1.4 & 24 \\
\hline $\mathrm{TL}_{16}$ & $\mathrm{TID}_{1}$ & 10 & 6.2 & -25.8 & 68.7 & 52.5 & 33.7 & 1.3 & 35 \\
\hline \multirow{2}{*}{$\mathrm{TL}_{17}$} & $\mathrm{TID}_{1}$ & 13 & 4.6 & 31.5 & -26.1 & 41.2 & -53.4 & 0.5 & 29 \\
\hline & $\mathrm{TID}_{2}$ & 4 & 3.3 & 31.5 & -26.1 & 59.6 & -50.0 & 0.9 & 54 \\
\hline $\mathrm{TL}_{18}$ & $\mathrm{TID}_{1}$ & 8 & 3.6 & 62.0 & -34.1 & 60.2 & -8.7 & 1.2 & 42 \\
\hline $\mathrm{TL}_{19}$ & $\mathrm{TID}_{1}$ & 9 & 4.7 & 48.8 & 48.3 & 71.2 & -5.2 & 1.1 & 39 \\
\hline \multirow{2}{*}{$\mathrm{TL}_{20}$} & $\mathrm{TID}_{1}$ & 6 & 6.1 & 86.2 & -12.4 & 87.4 & -21.0 & 0.8 & 43 \\
\hline & $\mathrm{TID}_{2}$ & 10 & 7.3 & 86.2 & -12.4 & 87.4 & 3.5 & 1.2 & 48 \\
\hline
\end{tabular}

Table 1 shows that 20 vehicles are conducting the delivery of tasks at time T. It also shows the current position, next destination, surplus weight and volume, cost per kilometer, fuel consumption per kilometer, and task lists of vehicles. The task lists on these vehicles in Table 3 show that 18 smart vehicles are transporting 22 tasks. $\mathrm{TID}_{1}$ and $\mathrm{TID}_{2}$ in Table 3 denote the tasks on the task lists. For example, $\mathrm{TL}_{1}$ has two tasks that are numbered as $\mathrm{TID}_{1}$ and $\mathrm{TID}_{2} . \mathrm{TL}_{2}$ has one task numbered as $\mathrm{TID}_{1}$. $\mathrm{VID}_{1}, \mathrm{VID}_{11}, \mathrm{VID}_{17}$, and $\mathrm{VID}_{20}$ have two tasks, respectively, while $\mathrm{VID}_{8}$ and $\mathrm{VID}_{12}$ do not have tasks. At this moment, 56 new tasks from different enterprises are submitted to DDC. Then, DDC publish these tasks among these vehicles in real time. Each vehicle obtains real-time information of these tasks, and selects the optimal tasks in a competitive manner. The optimization procedure of vehicles and tasks is introduced as follows.

Firstly, these vehicles and tasks are initialized to create the vehicle sets and task sets, then vehicle and task vectors are constructed. The circular region partition method proposed in Section 4 is used to select vehicles and tasks satisfying Constraint (3) as the pre-optimized subsets. Tasks in the circular regions created by information of vehicles are classified. The tasks in the intersections within more than one vehicle are re-optimized by the proposed method in Section 4. Finally, the optimal results meeting the optimization objective for new tasks and the tasks on the task lists of vehicles at time $\mathrm{T}$ are obtained as shown in Tables 4 and 5. Table 4 denotes the optimal results for 56 new tasks, and Table 5 denotes the re-optimized results for the tasks on the task lists of vehicles.

To demonstrate the optimization procedure of the proposed method, 20 smart vehicles and 78 tasks are simulated by applying the proposed optimization method in MATLAB as shown in Fig. 6. It should be noted that the center of Fig. 6, namely the point $(0,0)$, is the position of DDC. Fig. 6 (a) 
shows the optimal results obtained by IOMVT, in which blue, red, and green lines indicate real-time information of vehicles, the tasks on the task lists of vehicles, and new tasks. These lines also denote the vehicle vectors and task vectors. Blue lines indicate the vehicle vectors. Red and green lines denote task vectors for the tasks on the task lists of vehicles and new tasks, respectively. The center of a circular region created by a vehicle vector is the starting point of the vector that is numbered by vehicle No. (1-20 marked in blue), and the ending point of the vector is numbered by the value of the starting point plus the number of vehicles. For example, the starting and ending points of $\overrightarrow{\mathrm{VID}}_{1}$ are numbered by 1 and 21. The radius is the norm of the vector. The direction of the vector is the positive $\mathrm{X}$-axis $\left(\mathrm{X}^{+}\right)$. For a task vector, the starting point of the vector for new tasks is numbered by task No. (marked in red), while the ending point of the vector is not numbered. For example, the starting point of $\overrightarrow{\mathrm{TID}}_{1}$ is numbered by 1 . The tasks on the task lists of vehicles are not numbered because they are on the vehicles that are already numbered.

To describe the optimization process of IOMVT, $\mathrm{VID}_{4}$ and $\mathrm{VID}_{5}$ are taken as an example, and the results are shown in Fig. 6 (b). It is clear that $n=4, S=1, \operatorname{VID}_{4}$ and $\operatorname{VID}_{5} \in(n \geq 2, S=1)$, where $\mathrm{n}=4$ indicates $\mathrm{TID}_{1}, \mathrm{TID}_{13}, \mathrm{TID}_{23}$ and $\mathrm{TID}_{46}$. For the circular region created by $\mathrm{VID}_{4}, \mathrm{TID}_{23}$ belongs to Class 1 , and $\mathrm{TID}_{1}, \mathrm{TID}_{13}$, and $\mathrm{TID}_{46}$ belong to Class 2. For the circular region created by $\mathrm{VID}_{5}$, $\mathrm{TID}_{1}$, $\mathrm{TID}_{13}$, and $\mathrm{TID}_{23}$ belong to Class 1 , and $\mathrm{TID}_{46}$ belongs to Class 2 . The circular region partition method selects tasks for vehicles as shown in Fig. 6 (c). Firstly, VID 4 and VID Velect $_{5}$ tasks and 11 tasks, respectively, namely $\mathrm{n}=8$, and $\mathrm{n}=11$. For $\mathrm{VID}_{4}$, the tasks selected are $\mathrm{TID}_{1}, \mathrm{TID}_{5}, \mathrm{TID}_{13}$, $\mathrm{TID}_{23}, \mathrm{TID}_{24}, \mathrm{TID}_{25}, \mathrm{TID}_{45}$, and $\mathrm{TID}_{46}$. For VID 5 , the tasks selected include $\mathrm{TID}_{14}, \mathrm{TID}_{33}$, and $\mathrm{TID}_{34}$ besides tasks in the circular region created by $\mathrm{VID}_{4}$. Optimized by the proposed IOMVT, tasks meeting constraints of Eqs. (3) - (7) are $\mathrm{TID}_{23}, \mathrm{TID}_{24}$, and $\mathrm{TID}_{45}$ for $\mathrm{VID}_{4}$, and $\mathrm{TID}_{1}, \mathrm{TID}_{13}, \mathrm{TID}_{25}$, and $\mathrm{TID}_{46}$ for $\mathrm{VID}_{5}$. Red lines in Fig. 6 (c) indicate the distances from the midpoints of $\mid \overrightarrow{\mathrm{CP}_{4} \mathrm{ND}_{4} \mid}\left(\mathrm{VID}_{4}\right)$ and $\left|\overrightarrow{\mathrm{CP}_{5} \mathrm{ND}_{5}}\right|\left(\mathrm{VID}_{5}\right)$ to the midpoint of $\left|\overrightarrow{\mathrm{CP}_{\mathrm{j}} \mathrm{ND}_{\mathrm{j}}}\right|\left(\mathrm{TID}_{\mathrm{j}}\right)$.

Table 4 is the optimal results of IOMVT for vehicles and tasks. It shows the optimal tasks selected by vehicles, the effective distribution distance of tasks $\left(L_{j}\right)$, and the effective distribution distance of vehicles $\left(\mathrm{L}_{\mathrm{i}}\right)$. Here, to verify the efficiency of the proposed method on reducing the total distribution distance, the distance of vehicles $\left(\mathrm{L}_{\mathrm{i}}^{\prime}\right)$ obtained by $\mathrm{CM}$ is also included in Table 4 , and the current method will be introduced in the next section. Table 5 is the optimal results of IOMVT for the tasks on the task lists of vehicles. 


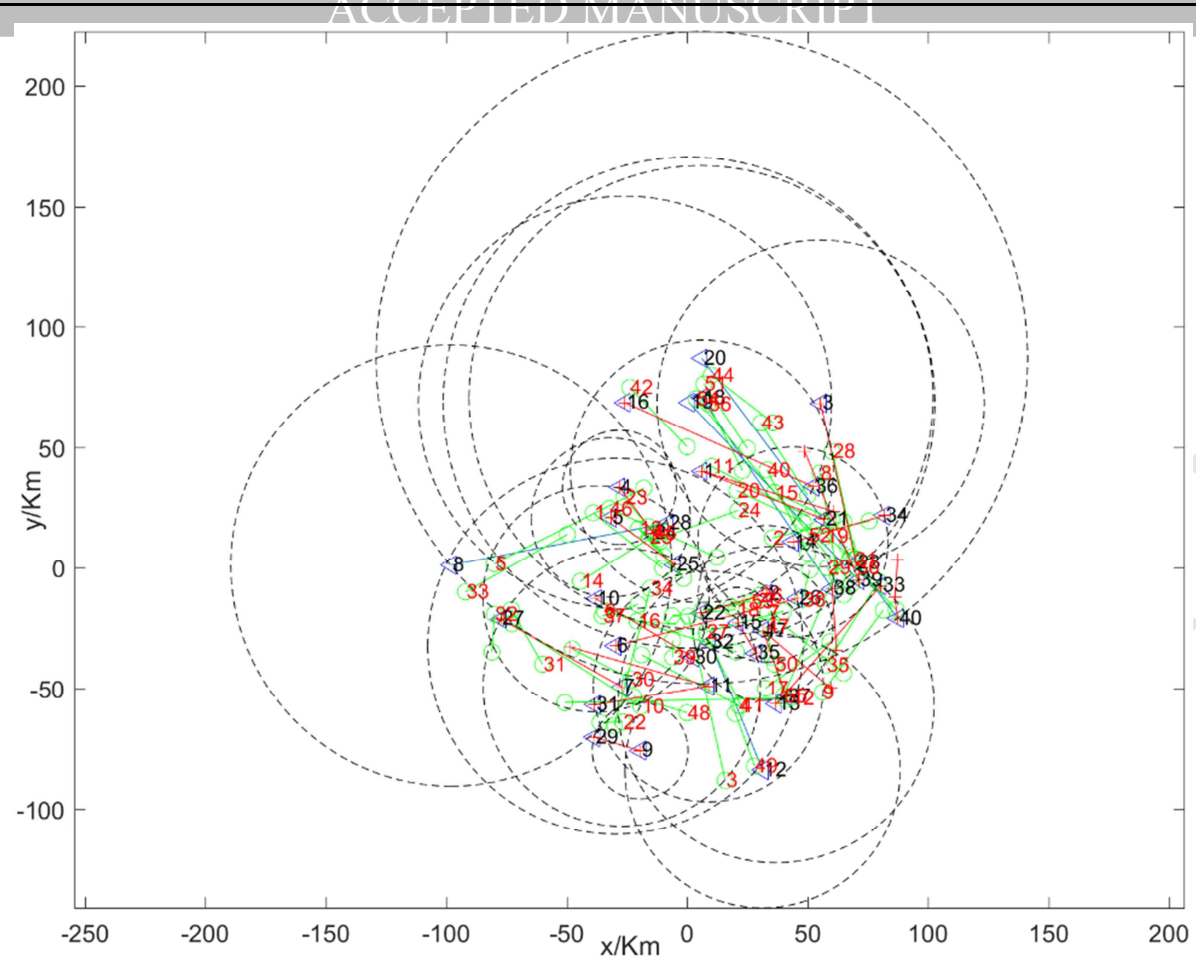

(a)

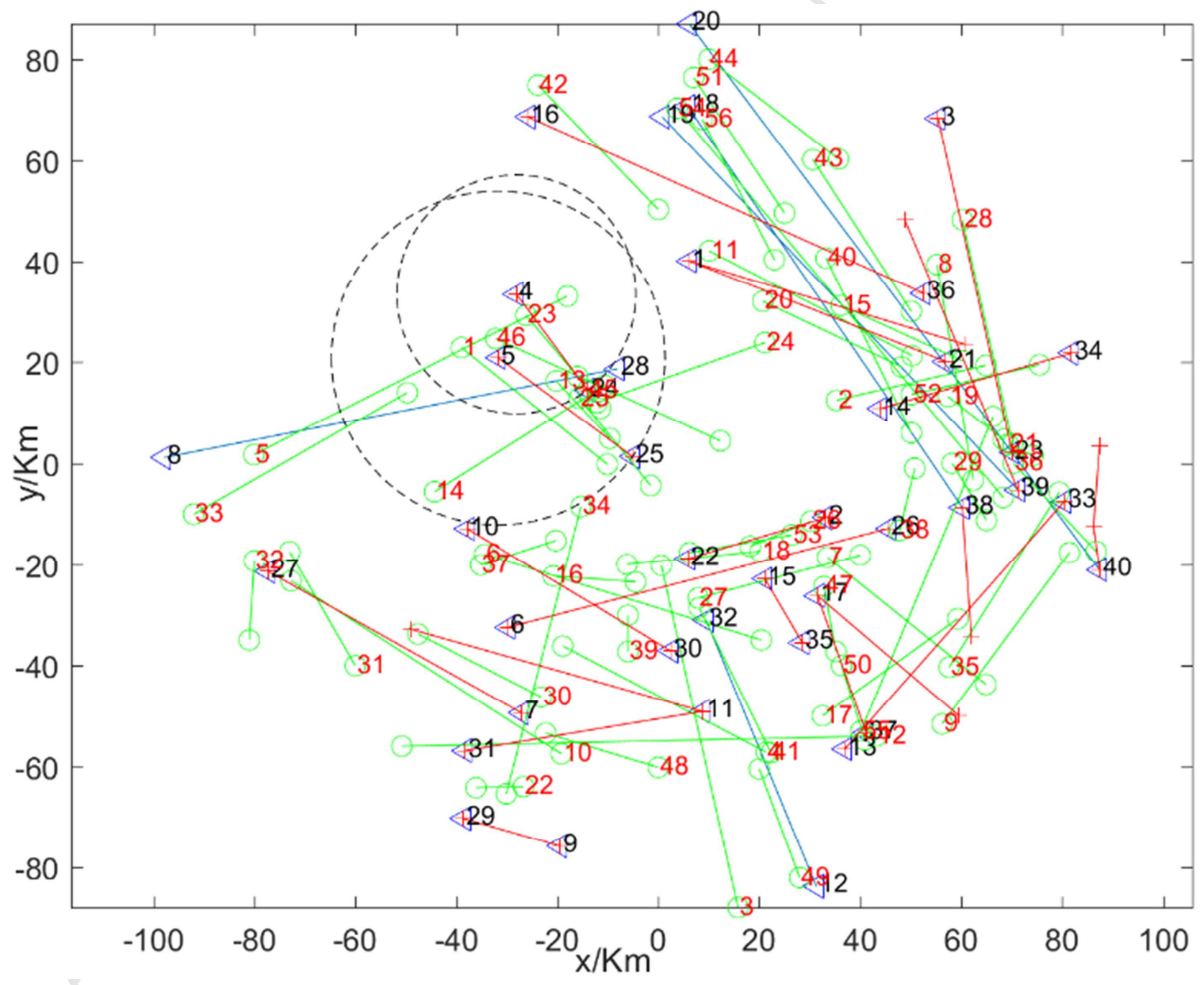

(b) 


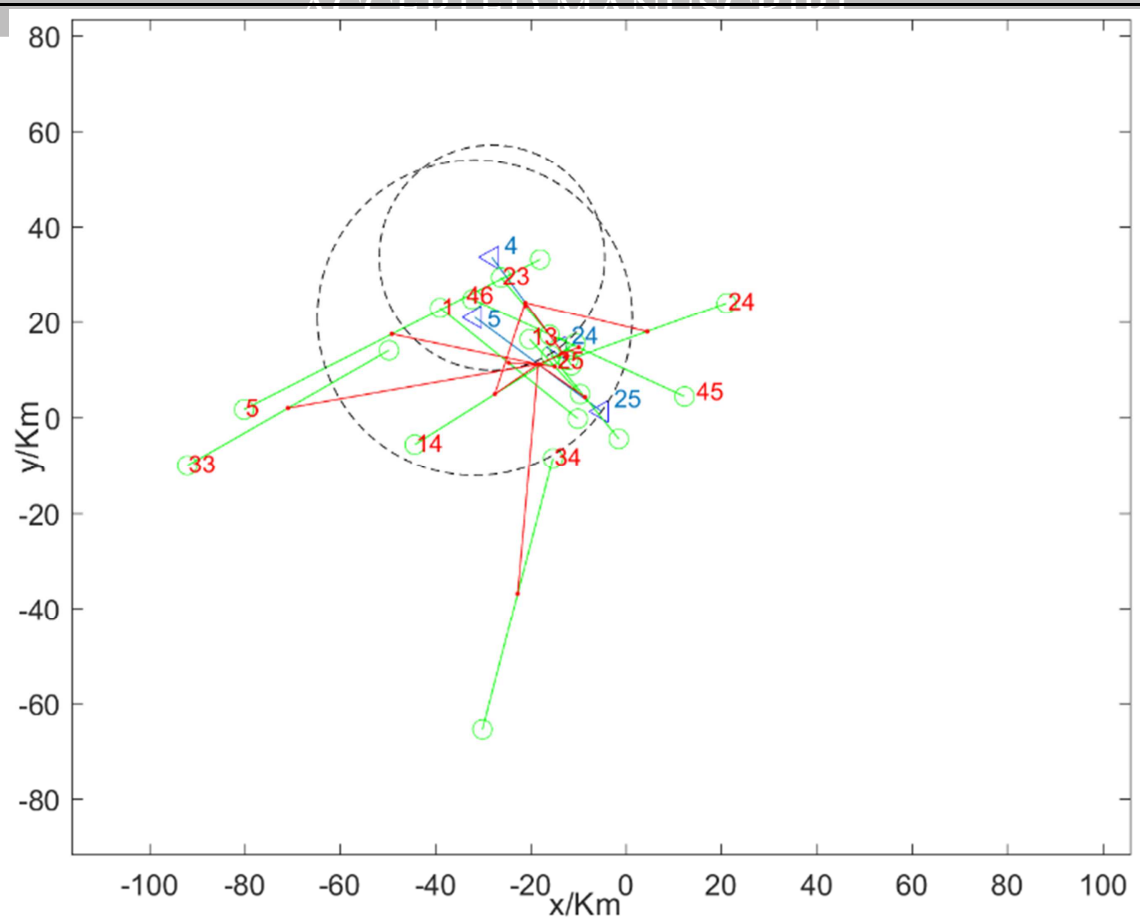

(c)

Fig. 6 (a) The optimal results obtained by IOMVT; (b) The optimization process of IOMVT for VID 4 and $\mathrm{VID}_{5}$; (c) The results of using IOMVT for $\mathrm{VID}_{4}$ and $\mathrm{VID}_{5}$.

Table 4 The optimal results of IOMVT for vehicles and tasks

\begin{tabular}{|c|c|c|c|}
\hline $\begin{array}{c}\text { Vehicle } \\
\text { No. }\end{array}$ & $\begin{array}{l}\text { Task } \\
\text { No. }\end{array}$ & $\mathrm{L}_{\mathrm{j}}(\mathrm{Km})$ & $\mathrm{L}_{\mathrm{i}} / \mathrm{L}_{\mathrm{i}}^{\prime}(\mathrm{Km})$ \\
\hline \multirow{3}{*}{$\mathrm{VID}_{1}$} & $\mathrm{TID}_{11}$ & 69.26 & \multirow{3}{*}{$76.78 / 264.54$} \\
\hline & $\mathrm{TID}_{15}$ & 29.18 & \\
\hline & $\mathrm{TID}_{20}$ & 57.73 & \\
\hline \multirow{3}{*}{$\mathrm{VID}_{2}$} & $\mathrm{TID}_{18}$ & 27.69 & \multirow{3}{*}{$42.32 / 136.27$} \\
\hline & $\mathrm{TID}_{26}$ & 13.87 & \\
\hline & $\mathrm{TID}_{53}$ & 21.28 & \\
\hline \multirow{4}{*}{$\mathrm{VID}_{3}$} & $\mathrm{TID}_{8}$ & 43.53 & \multirow{4}{*}{$93.87 / 280.55$} \\
\hline & $\mathrm{TID}_{19}$ & 27.18 & \\
\hline & $\mathrm{TID}_{21}$ & 8.11 & \\
\hline & $\mathrm{TID}_{28}$ & 68.59 & \\
\hline \multirow{3}{*}{$\mathrm{VID}_{4}$} & $\mathrm{TID}_{23}$ & 15.83 & \multirow{3}{*}{$98.51 / 136.74$} \\
\hline & $\mathrm{TID}_{24}$ & 70.27 & \\
\hline & $\mathrm{TID}_{45}$ & 4.44 & \\
\hline \multirow{4}{*}{$\mathrm{VID}_{5}$} & $\mathrm{TID}_{1}$ & 42.51 & \multirow{4}{*}{$78.40 / 275.06$} \\
\hline & $\mathrm{TID}_{13}$ & 15.70 & \\
\hline & $\mathrm{TID}_{25}$ & 27.15 & \\
\hline & $\mathrm{TID}_{46}$ & 63.80 & \\
\hline \multirow{3}{*}{$\mathrm{VID}_{6}$} & $\mathrm{TID}_{16}$ & 18.95 & \multirow{3}{*}{$98.47 / 229.10$} \\
\hline & $\mathrm{TID}_{27}$ & 67.57 & \\
\hline & $\mathrm{TID}_{38}$ & 80.03 & \\
\hline \multirow{4}{*}{$\mathrm{VID}_{7}$} & $\mathrm{TID}_{10}$ & 148.47 & \multirow{4}{*}{$263.94 / 329.34$} \\
\hline & $\mathrm{TID}_{30}$ & 26.87 & \\
\hline & $\mathrm{TID}_{31}$ & 59.09 & \\
\hline & $\mathrm{TID}_{32}$ & 76.30 & \\
\hline $\mathrm{VID}_{8}$ & $\mathrm{TID}_{5}$ & 114.45 & $141.28 / 249.66$ \\
\hline
\end{tabular}

\begin{tabular}{|c|c|c|c|}
\hline \multirow{3}{*}{$\mathrm{VID}_{9}$} & $\mathrm{TID}_{14}$ & 39.71 & \\
\hline & $\mathrm{TID}_{33}$ & 49.48 & \\
\hline & $\mathrm{TID}_{34}$ & 75.86 & $143.29 / 144.72$ \\
\hline \multirow{3}{*}{$\mathrm{VID}_{10}$} & $\mathrm{TID}_{6}$ & 57.38 & \multirow{3}{*}{$143.57 / 210.07$} \\
\hline & $\mathrm{TID}_{37}$ & 136.01 & \\
\hline & $\mathrm{TID}_{39}$ & 7.20 & \\
\hline \multirow{2}{*}{$\mathrm{VID}_{11}$} & $\mathrm{TID}_{22}$ & 9.40 & \multirow{2}{*}{$92.31 / 164.28$} \\
\hline & $\mathrm{TID}_{48}$ & 23.31 & \\
\hline \multirow{4}{*}{$\mathrm{VID}_{12}$} & $\mathrm{TID}_{3}$ & 103.38 & \multirow{4}{*}{$131.90 / 276.44$} \\
\hline & $\mathrm{TID}_{4}$ & 45.49 & \\
\hline & $\mathrm{TID}_{41}$ & 82.88 & \\
\hline & $\mathrm{TID}_{49}$ & 41.37 & \\
\hline \multirow{4}{*}{$\mathrm{VID}_{13}$} & $\mathrm{TID}_{9}$ & 42.32 & \multirow{4}{*}{$307.81 / 428.97$} \\
\hline & $\mathrm{TID}_{17}$ & 33.03 & \\
\hline & $\mathrm{TID}_{35}$ & 65.92 & \\
\hline & $\mathrm{TID}_{55}$ & 109.16 & \\
\hline \multirow{2}{*}{$\mathrm{VID}_{14}$} & $\mathrm{TID}_{2}$ & 64.89 & \multirow{2}{*}{$73.66 / 159.25$} \\
\hline & $\mathrm{TID}_{52}$ & 39.45 & \\
\hline $\mathrm{VID}_{15}$ & $\mathrm{TID}_{7}$ & 54.96 & $68.08 / 72.50$ \\
\hline \multirow{2}{*}{$\mathrm{VID}_{16}$} & $\mathrm{TID}_{42}$ & 53.11 & \multirow{2}{*}{$115.01 / 257.99$} \\
\hline & $\mathrm{TID}_{56}$ & 44.94 & \\
\hline \multirow{3}{*}{$\mathrm{VID}_{17}$} & $\mathrm{TID}_{12}$ & 94.14 & \multirow{3}{*}{$162.35 / 221.10$} \\
\hline & $\mathrm{TID}_{47}$ & 13.12 & \\
\hline & $\mathrm{TID}_{50}$ & 52.30 & \\
\hline \multirow{3}{*}{$\mathrm{VID}_{18}$} & $\mathrm{TID}_{29}$ & 57.41 & \multirow{3}{*}{$161.79 / 160.63$} \\
\hline & $\mathrm{TID}_{40}$ & 38.60 & \\
\hline & $\mathrm{TID}_{51}$ & 38.19 & \\
\hline $\mathrm{VID}_{19}$ & $\mathrm{TID}_{54}$ & 114.85 & $114.85 / 220.30$ \\
\hline
\end{tabular}


Table 5 The optimal results of IOMVT for tasks on the task lists of vehicles

\begin{tabular}{|c|c|c|c|c|}
\hline $\begin{array}{c}\text { TL } \\
\text { No. }\end{array}$ & $\begin{array}{l}\text { Task } \\
\text { No. }\end{array}$ & $\mathrm{L}_{\mathrm{j}} / \mathrm{Km}$ & $\mathrm{L}_{\mathrm{j}}^{\prime} / \mathrm{Km}$ & $\mathrm{t}_{\mathrm{j}} /$ day \\
\hline \multirow{2}{*}{$\mathrm{TL}_{1}$} & $\mathrm{TID}_{1}$ & 67.0 & 54.6 & 0.2 \\
\hline & $\mathrm{TID}_{2}$ & 60.5 & 57.3 & 0.1 \\
\hline $\mathrm{TL}_{2}$ & $\mathrm{TID}_{1}$ & 30.2 & 28.4 & 0.1 \\
\hline $\mathrm{TL}_{3}$ & $\mathrm{TID}_{1}$ & 87.6 & 67.9 & 0.3 \\
\hline $\mathrm{TL}_{4}$ & $\mathrm{TID}_{1}$ & 23.8 & 23.7 & 0.0 \\
\hline $\mathrm{TL}_{5}$ & $\mathrm{TID}_{1}$ & 55.3 & 33.0 & 0.7 \\
\hline $\mathrm{TL}_{6}$ & $\mathrm{TID}_{1}$ & 83.7 & 77.9 & 0.1 \\
\hline $\mathrm{TL}_{7}$ & $\mathrm{TID}_{1}$ & 155.2 & 57.5 & 1.7 \\
\hline $\mathrm{TL}_{9}$ & $\mathrm{TID}_{1}$ & 133.5 & 19.9 & 5.7 \\
\hline $\mathrm{TL}_{10}$ & $\mathrm{TID}_{1}$ & 112.0 & 47.0 & 1.4 \\
\hline
\end{tabular}

\begin{tabular}{ccccc}
\hline \multirow{2}{*}{$\mathrm{TL}_{11}$} & $\mathrm{TID}_{1}$ & 66.0 & 47.7 & 0.4 \\
\cline { 2 - 5 } & $\mathrm{TID}_{2}$ & 92.3 & 60.0 & 0.5 \\
\hline $\mathrm{TL}_{13}$ & $\mathrm{TID}_{1}$ & 114.6 & 65.6 & 0.7 \\
\hline $\mathrm{TL}_{14}$ & $\mathrm{TID}_{1}$ & 56.4 & 39.5 & 0.4 \\
\hline $\mathrm{TL}_{15}$ & $\mathrm{TID}_{1}$ & 30.7 & 14.6 & 1.1 \\
\hline $\mathrm{TL}_{16}$ & $\mathrm{TID}_{1}$ & 115.0 & 85.8 & 0.3 \\
\hline \multirow{2}{*}{$\mathrm{TL}_{17}$} & $\mathrm{TID}_{1}$ & 32.6 & 29.0 & 0.1 \\
\cline { 2 - 5 } & $\mathrm{TID}_{2}$ & 51.3 & 36.9 & 0.4 \\
\hline $\mathrm{TL}_{18}$ & $\mathrm{TID}_{1}$ & 28.5 & 25.5 & 0.1 \\
\hline $\mathrm{TL}_{19}$ & $\mathrm{TID}_{1}$ & 58.0 & 58.0 & 0 \\
\hline \multirow{2}{*}{$\mathrm{TL}_{20}$} & $\mathrm{TID}_{1}$ & 8.7 & 8.7 & 0.0 \\
\cline { 2 - 5 } & $\mathrm{TID}_{2}$ & 33.2 & 16.0 & 1.1
\end{tabular}

\subsection{Analysis of optimal results between IOMVT and current method (CM)}

To demonstrate the effectiveness of the proposed method, the same tasks in Table 2 are optimized by using CM. Currently, a centralized and one-way logistics service pattern is widely used by logistics companies in practice. This method in practice is defined as the current method in this paper. The optimal results for two methods are shown in Table 6.

Table 6 The optimal results of IOMVT and CM

\begin{tabular}{ccccccccc}
\hline & $\mathrm{L} / \mathrm{Km}$ & $\mathrm{C} / \$$ & $\mathrm{TFC} / \mathrm{L}$ & $\mathrm{R}^{\prime}=\left(\mathrm{V}^{\prime}, \mathrm{W}^{\prime}\right)$ & $\overline{\mathrm{FC}}$ & $\mathrm{N}$ & $\overline{\mathrm{R}}$ & $\mathrm{C}^{\prime}$ \\
\hline IOMVT & 2573.01 & 5904.90 & 814.83 & $(0.3896,0.4170)$ & 0.3167 & 20 & 0.7368 & 0.3598 \\
$\mathrm{CM}$ & 4397.27 & 9223.74 & 1344.01 & $(0.1733,0.1781)$ & 0.3046 & 76 & & \\
\hline
\end{tabular}

L, C, and TFC of IOMVT are 2573.01, 5904.90, and 814.83, respectively. By contrast, those of $\mathrm{CM}$ are 4397.27, 9223.74, and 1344.201, respectively. They are decreased by $41.49 \%, 35.98 \%$, and $39.37 \%$, compared with the results of CM. It is clear that IOMVT has significant advantages on the routing optimization for vehicles and tasks, and reducing the total distribution distance, the total cost, and the total fuel consumption. In addition, $V^{\prime}$ and $W^{\prime}$ of IOMVT are $38.96 \%$ and $41.70 \%$, respectively, by contrast, those of $\mathrm{CM}$ are $14.33 \%$, and $17.81 \%$, respectively. Therefore, the average loading rate of vehicles ( $\mathrm{R}^{\prime}$ ) obtained by IOMVT is better than that of CM. It can be concluded that IOMVT can effectively increase the average effective loading rate of vehicles and the average effective weight of vehicles. The average fuel consumption per kilometer $(\overline{\mathrm{FC}})$ of IOMVT $(0.3167)$ is larger than that of $\mathrm{CM}$ (0.3046). This is because the increase in loading weight of vehicles leads to the increase in fuel consumption per kilometer. However, the increase of fuel consumption per kilometer (3.9\%) is very small, compared with near $25 \%$ of the increase in fuel consumption from the no-load to the full-load in the round-trip. The number of vehicles used for IOMVT is 20 , while it is 76 for CM. This is because $\mathrm{CM}$ in the logistics activities is a centralized and one-way logistics service, and finishing 56 new tasks $\left(\mathrm{TID}_{1}-\mathrm{TID}_{56}\right)$ needs 56 times of logistics delivery. Therefore, the number of vehicles used to finish the same number of tasks in the logistics activities is reduced significantly. The total cost in this paper does not consider the cost of increasing vehicles, such as the rental fees of vehicles. The rate of saving cost and the utilization rate of vehicles $\left(C^{\prime}, \bar{R}\right)$ are $35.98 \%$ and $72.68 \%$, respectively. As a result, the efficiency of IOMVT in reducing the total cost, the total distance, the total fuel consumption, and increasing the utilization rate of vehicles can be verified. The reduction in the total cost, the fuel consumption, the total distribution distance can decrease logistics costs and the greenhouse emission 

activities and the increase in the utilization rate of vehicles can contribute to improving social issues like the burden of the traffic in cities. It can be concluded that this research enables the current logistics practices towards green logistics and develops a sustainable balance between economic, environmental and social objectives.

It can be found that the proposed IoT-enabled dynamic optimization method in this paper implements the optimal management and allocation of logistics resources and the real-time response and optimization of new tasks. The efficiency of the real-time information enabled circular region partition method in the mapping and pre-optimization of vehicles and tasks is verified. The circular region partition method based on the theory of the circle inscribed triangle can effectively decrease the complexity of optimization for tasks and vehicles. Vehicles in the circular regions actively selecting the optimal tasks in a competitive way is a novel logistics service pattern which better improves the efficiency of logistics services. It also solves some of the typical challenges existing in the current logistics practice. In addition, the logistics resources registry and integration in EIS enhances the utilization and sharing efficiency of logistics resources. The real-time information enabled routing optimization and navigation services, and loading services developed in this paper improves the efficiency of logistics services and the utilization rates of vehicles, and avoids the errors in loading/unloading tasks. It can be concluded that this paper provides a new direction and strategy towards low-carbon and green logistics, and adapts to the trend of development of the logistics industry in the future.

It can be seen from Table 5 that the effective distribution distance of tasks on the task lists of vehicles optimized by the proposed method increases because of accepting new tasks. Finishing new tasks and the tasks on the task lists of vehicles leads to the change of the delivery schemes for the tasks on the task lists. This also results in the delivery delay for the tasks on the task lists except for tasks $\mathrm{TID}_{1}$ on $\mathrm{TL}_{4}, \mathrm{TL}_{19}$, and $\mathrm{TL}_{20}$. In addition, even though the delay of some tasks is in the range of the allowed time of delay, this may affect customer satisfaction. The influence of delivery delay can be reflected by the quality of services as shown in Fig. 5. Most importantly, the key performance indicators of the proposed IOMVT such as the total cost, the total distance, the total fuel consumption, the average loading rate, and the number of vehicles used are better than those of CM.

There are two main differences between the proposed IoT-enabled dynamic optimization method for vehicles and tasks and the current method widely used in current logistics activities. Firstly, for the current method, it is a centralized and one-way logistics service pattern and tasks are only assigned based on the destination of tasks in a certain period. It lacks the real-time information of logistics resources and new tasks cannot be sensed and handled timely. These cases will lead to the increase of logistics costs, the waste of logistics resources, and the low loading rate of vehicles. While IoT is applied in the proposed dynamic optimization method for vehicles and tasks. Under the constructed IoMT environment, the real-time information of logistics resources can be sensed and collected, and real-time optimization and decision-making can be achieved. Secondly, vehicles in CM is only responsible for transporting the tasks, and do not have the ability of "thinking". Tasks are intensively assigned based on their destinations in a logistics service point. Therefore, new tasks cannot be handled in real time. For the IoT-enabled dynamic optimization method, vehicles have the capacity of sensing and obtaining real-time information of logistics resources, and vehicles can actively select the optimal tasks in a competitive way. Thus, new tasks can be selected by smart vehicles that are in the ongoing 

services, and achieve the optimal allocation of logistics resources. In this case, urgent tasks can also be optimized and assigned timely.

\section{Conclusions and future works}

This paper proposes an IoT-enabled dynamic optimization method for smart vehicles and logistics tasks. Through using IoT technology, a real-time information-driven dynamic optimization strategy effectively improves vehicles' utilization rate and reduces the cost, the fuel consumption, and the number of vehicles used, and provides the optimal routing with ensured efficiency of logistics services. It provides a new direction and strategy towards low-carbon and green logistics, and adapts to the trend of logistics industry in the future.

There are three contributions in this paper. The first is the real-time information sensing model for logistics resources. Intelligent identification devices are selected and configured to construct the IoTbased sensing environment among vehicles, tasks, and enterprises, then real-time, accurate, and valueadded information of logistics resources can be sensed and collected timely and shared among logistics enterprises. The second is that the optimal management and allocation of logistics resources based on EIS is developed to achieve integration, management, sharing, and allocation of logistics resources. Besides, real-time information enabled routing optimization and navigation services, and loading services of tasks are developed to improve the efficiency of logistics and the utilization rates of vehicles, and avoid the errors in loading/unloading tasks. The third is the IoT-enabled dynamic optimization method for vehicles and tasks. A real-time information enabled circular region partition method is developed to achieve optimization and mapping between vehicles and tasks. The objective function for optimizing vehicles and tasks is formulated, and the theory of the circle inscribed triangle is used to obtain the optimal distribution results. The results show that IOMVT contributes to improved efficiency of logistics and the utilization rate of vehicles, reduced logistics cost, and achieving sustainable logistics services.

Future research will focus on the optimization of the global logistics network of logistics resources, and the optimal management and allocation of logistics resources in the global logistics network. This is an upcoming challenge faced by the logistics industry with the rapid increase of logistics demands. The global logistics network is a complex network of the logistics system with many network nodes. How to obtain the global optimal results developed from the local optimal strategies for the entire logistics supply chain is worth studying. This involves in the design of the optimization methods and strategies and the sharing of real-time information among all the network nodes.

\section{Acknowledgments}

The authors gratefully acknowledge the financial supports of National Science Foundation of China (51675441, 51475096), the 111 Project Grant (B13044), and the Fundamental Research Funds for the Central Universities (3102017jc04001). This research is also supported in part by the Circularis

(Circular Economy through Innovating Design) project funded by Vinnova - Sweden's innovation 
agency (2016-03267) and the Simon (New Application of AI for Services in Maintenance towards a

Circular Economy) project funded by Vinnova - Sweden's innovation agency (2017-01649).

\section{References}

Angeles, R., 2006. RFID technologies: Supply-chain applications and implementation issues. Inform. Syst. Manage. 22 (1), 51-65.

Braekers, K., Ramaekers, K., Van Nieuwenhuyse, I., 2016. The vehicle routing problem: state of the art classification and review. Comput. Ind. Eng. 99, 300-313.

Bramel, J., Simchi-Levi, D., 1995. A location based heuristic for general routing problems. Oper. Res. 43, 649-660.

Cattaruzza, D., Absi, N., Feillet, D., González-Feliu, J., 2017. Vehicle routing problems for city logistics. Euro. J. Transp. Logist. 6(1), 51-79.

Chen, H.K., Hsueh, C.F., Chang, M.S., 2006. The real-time time-dependent vehicle routing problem. Transport. Res. E-Log. 42 (5), 383-408.

Chow, J.Y.J., Ritchie, S.G., Jeong, K., 2014. Nonlinear inverse optimization for parameter estimation of commodity-vehicle-decoupled freight assignment. Transport. Res. E-Log. 67, 71-91.

Chhabra, D., Garg, S. K., Singh, R. K., 2017. Analyzing alternatives for green logistics in an Indian automotive organization: A case study. J. Clean. Prod. 167, 962-969.

Crainic, T.G., Ricciardi, N., Storchi, G. 2004. Advanced freight transportation systems for congested urban areas. Transport. Res. C-Emer. 12(2), 119-137.

Dekker, R., Bloemhof, J., Mallidis, I., 2012. Operations Research for green logistics - An overview of aspects, issues, contributions and challenges. Eur. J. Oper. Res. 219 (6), 671-679.

European Ecommerce Report 2017. https://www.ecommerce-europe.eu/.

Fahimnia, B., Bell, M. G., Hensher, D.A., Sarkis, J. (Eds.)., 2015. Green logistics and transportation: A sustainable supply chain perspective. Springer.

Fahimnia, B., Sarkis, J., Davarzani, H., 2015. Green supply chain management: A review and bibliometric analysis. Int. J. Prod. Econ. 162, 101-114.

Ghiani, G., Guerriero, F., Laporte, G., Musmanno, R., 2003. Real-time vehicle routing: Solution concepts, algorithms and parallel computing strategies. Eur. J. Oper. Res. 151 (1), 1-11.

Green logistics, 2010. http://www.greenlogistics.org/.

He, Z., Chen, P., Liu, H., Guo, Z., 2017. Performance measurement system and strategies for developing low-carbon logistics: A case study in China J. Clean. Prod. 156, 395-405.

Isaksson, K., Bjorklund, M., Evangelista, P., Huge-Brodin, M., 2011. The challenge and adoption of green initiatives for transport and logistics service providers. In: The 16th Annual LRN Conference 7-9 September, Southampton, United Kingdom, 2011, 1-10.

Khan, S.A.R., Zhang, Y., Anees, M., Golpîra, H., Lahmar, A., Qianli, D., 2018. Green supply chain management, economic growth and environment: A GMM based evidence. J. Clean. Prod. 185, 588-599.

Kim, G., Ong, Y.S., Heng, C.K., Tan, P.S., Zhang, N.A., 2015. City vehicle routing problem (city VRP): A review. IEEE T. Intell. Transp. 16 (4), 1654-1666.

Koh, S.L., Wang, L., 2010. Overview of enterprise networks and logistics for agile manufacturing. In 
Lai, K., Wong, C.W.Y., 2012. Green logistics management and performance: Some empirical evidence from Chinese manufacturing exporters. Omega. 40 (3), 267-282.

Langevin, A., Mbaraga, P., Campbell, J.F., 1996. Continuous approximation models in freight distribution: an overview. Transp. Res. Part B Methodol. 30 (3), 163-188.

Lee, H.L., So, K.C., Tang, C.S., 2000. The value of information sharing in a two-level supply chain. Manage. Sci. 46 (5), 626-643.

Li, X., Li, D., Wan, J., Vasilakos, A.V., Lai, C.F., Wang, S., 2017. A review of industrial wireless networks in the context of industry 4.0. Wirel. Netw. 23(1), 23-41.

Liao, T.Y., Hu, T.Y., 2011. An object-oriented evaluation framework for dynamic vehicle routing problems under real-time information. Expert. Syst. Appl. 38 (10), 12548-12558.

Liu, S., Zhang, G., Wang, L., 2018. IoT-enabled dynamic optimisation for sustainable reverse logistics. Proc. CIRP, 69(1), 662-667.

Marinakis, Y., Marinaki, M., 2010. A hybrid genetic-Particle Swarm Optimization Algorithm for the vehicle routing problem. Expert. Syst. Appl. 37 (2), 1446-1455.

Martel, A., Klibi, W., 2016. Supply chains: issues and opportunities, In: Designing Value-creating Supply Chain Networks. Springer International Publishing, 1-43, 2016.

Masson, R., Trentini, A., Lehuédé, F., Malhéné, N., Péton, O., Tlahig, H., 2017. Optimization of a city logistics transportation system with mixed passengers and goods. Euro. J. Transp. Logist. 6(1), 81109.

McKinnon, A., 2010. The role of government in promoting green logistics. Green logistics: improving the environmental sustainability of logistics. Kogan Page, London, 341-360. 2010

McKinnon, A., Edwards, J., 2010. Opportunities for improving vehicle utilization. Green Logistics: Improving the Environmental Sustainability of Logistics, 195-213.

McKinnon, A., Browne, M., Whiteing, A., Piecyk, M., 2015. Green logistics: improving the environmental sustainability of logistics. Kogan Page Publishers.

Muñoz-Villamizar, A., Santos, J., Montoya-Torres, J.R., Jaca, C., 2018. Using OEE to evaluate the effectiveness of urban freight transportation systems: A case study. Int. J. Prod. Econ. 197, 232-242.

Niu, Y., Yang, Z., Chen, P., Xiao, J., 2018. Optimizing the green open vehicle routing problem with time windows by minimizing comprehensive routing cost. J. Clean. Prod. 171, 962-971.

Nativi, J.J., Lee, S., 2012. Impact of RFID inform-sharing strategies on a decentralized supply chain with reverse logistics operations. Int. J. Prod. Econ. 136 (2), 366-377.

Okulewicz, M., Mańdziuk, J., 2017. The impact of particular components of the PSO-based algorithm solving the Dynamic Vehicle Routing Problem. Appl. Soft. Comput. 58, 586-604.

Pålsson, H., Pettersson, F., Hiselius, L.W., 2017. Energy consumption in e-commerce versus conventional trade channels-Insights into packaging, the last mile, unsold products and product returns. J. Clean. Prod. 164, 765-778.

Pavone, M., Frazzoli, E., Bullo, F., 2011. Adaptive and distributed algorithms for vehicle RFIDrouting in a stochastic and dynamic environment. IEEE T. Automat. Contr. 56 (6), 1259-1274.

Poon, T.C., Choy, K.L., Chow, H.K.H., Lau, H.C.W., Chan, F.T.S., Ho, K.C., 2009. A RFID casebased logistics resource management system for managing order-picking operations in warehouses. Expert. Syst. Appl. 36 (4), 8277-8301.

Powell, W.B., 1986. A stochastic model of the dynamic vehicle allocation problem. Transport. Sci. 42, 
Qu, T., Lei, S.P., Wang, Z.Z., Nie, D.X., Chen, X., Huang, G.Q., 2015. IoT-based real-time production logistics synchronization system under intelligent cloud manufacturing. Int. J. Adv. Manuf. Tech. 84(1), 147-164.

Rehman, M.A., Seth, D., Shrivastava, R.L., 2016. Impact of green manufacturing practices on organizational performance in Indian context: an empirical study. J. Clean. Prod. 137, 427-448.

Rao, P., Holt, D., 2005. Do green supply chains lead to competitiveness and economic performance? Int. J. Op. Prod. Manag. 25(9), 898-916.

Rose, L.A., Chew, B.C., Hamid, M.S.R.B.A., 2018. Green Logistics Implementation in Malaysian Logistics Industry. Adv. Sci. Lett. 24(6), 4214-4217.

Ross, D.F., 2003. Introduction to e-supply chain management: Engaging technology to build marketwinning business partnership, St. Lucie Press, 2003.

Ruan, J., Shi, Y., 2016. Monitoring and assessing fruit freshness in IoT-based e-commerce delivery using scenario analysis and interval number approaches. Inform. Sciences. 373, 557-570.

Sarkis, J., 2003. A strategic decision framework for green supply chain management. J. Clean. Prod. 11 (4), 397-409.

Savelsbergh, M., Van Woensel, T., 2016. 50th anniversary invited article-city logistics: Challenges and opportunities. Transport. Sci. 50(2), 579-590.

Sheng, Z., Yang, S., Yu, Y., Vasilakos, A., Mccann, J., Leung, K., 2013. A survey on the IETF protocol suite for the internet of things: Standards, challenges, and opportunities. IEEE Wirel. Commun. 20 (6), 91-98.

Sheu, J.B., 2006. A novel dynamic resource allocation model for demand-responsive city logistics distribution operations. Transport. Res. E-Log. 42, 445-472.

Soleimani, H., Chaharlang, Y., Ghaderi, H., 2017. Collection and distribution of returnedremanufactured products in a vehicle routing problem with pickup and delivery considering sustainable and green criteria. J. Clean. Prod. 172, 960-970.

Srivastava, S.K., 2007. Green supply-chain management: a state-of-the-art literature review. Int. J. Manag. Rev. 9 (1), 53-80.

Toth, P., Vigo, D., 2014. Vehicle routing: problems, methods, and applications. Society for Industrial and Applied Mathematics, 2014.

Vieira, J.G.V., Fransoo, J.C., Carvalho, C.D., 2015. Freight distribution in megacities: perspectives of shippers, logistics service providers and carriers. J. Transp. Geogr., 46, 46-54.

Vukadinović, K., Teodorović, D., Pavković, G. 1999. An application of neuro-fuzzy modeling: The vehicle assignment problem. Eur. J. Oper. Res. 114 (3), 474-488.

Wang, L., Koh, S.L., (Eds.), 2010. Enterprise networks and logistics for agile manufacturing, SpringerVerlag, London, UK, 406.

Wang, L., Givehchi, M., Adamson, G., Holm, M., 2011. A sensor-driven 3D model-based approach to remote real-time monitoring. CIRP Ann-Manuf. Techn. 60 (1), 493-496.

Wang, X., Ruan, J., Shi, Y., 2012. A recovery model for combinational disruptions in logistics delivery: Considering the real-world participators. Int. J. Prod. Econ. 140 (1), 508-520.

Pillac, V., Gendreau, M., Guéret, C., Medaglia, A.L., 2013. A review of dynamic vehicle routing problems. Eur. J. Oper. Res. 225 (1), 1-11.

Want, R., 2006. An introduction to RFID technology. IEEE Pervas. Comput. 5(1), 25-33. 
Yang, J., Guo, J., Ma, S., 2016. Low-carbon city logistics distribution network design with resource deployment. J. Clean. Prod. 119, 223-228.

Yu, B., Yang, Z.Z., Yao, B., 2009. An improved ant colony optimization for vehicle routing problem. Eur. J. Oper. Res. 196 (1), 171-176.

Zaman, K., Shamsuddin, S., 2017. Green logistics and national scale economic indicators: evidence from a panel of selected European countries. J. Clean. Prod. 143, 51-63.

Zhang, Y., Zhang, G., Wang, J., Sun, S., Si, S., Yang, T., 2015. Real-time information capturing and integration framework of the internet of manufacturing things. Int. J. Comput. Integ. M. 28 (8), 811-822.

Zhang, Y., Liu, S., Liu, Y., Li, R., 2016. Smart box-enabled product-service system for cloud logistics. Int. J. Prod. Res. 54 (22), 6693-6706.

Zhang, Y., Wang, J., Liu, Y., 2017a. Game theory based real-time multi-objective flexible job shop scheduling considering environmental impact. J. Clean. Prod. 167, 665-679.

Zhang, Y., Zhu, Z., Lv, J., 2017b. CPS-Based smart control model for shopfloor material handling. IEEE T. Ind. Inform. 14(4),1764-75.

Zhang, Y., Ren, S., Liu, Y., Sakao, T., Huisingh, D., 2017c. A framework for Big Data driven product lifecycle management. J. Clean. Prod. 159, 229-240.

Zhang, Y.F., Guo, Z.G., Lv, J.X., Liu, Y., 2018a. A Framework for Smart Production-Logistics Systems based on CPS and Industrial IoT. IEEE T. Ind. Inform. 14 (9), 4019-4032.

Zhang, Y., Liu, S., Liu, Y., Yang, H., Li, M., Huisingh, D., Wang, L., 2018b. The 'Internet of Things' enabled real-time scheduling for remanufacturing of automobile engines. J. Clean. Prod. 185, 562575.

Ren S, Zhang Y, Liu Y, Sakao T, Huisingh D, Almeida CM., 2018c. A comprehensive review of big data analytics throughout product lifecycle to support sustainable smart manufacturing: a framework, challenges and future research directions. J. Clean. Prod. Accepted. 10.1016/j.jclepro.2018.11.025 